\title{
Mass limits for dark clusters of degenerate fermions
}

\author{
M. Membrado ${ }^{1}$ and A. F. Pacheco ${ }^{1,2}$ \\ 1 Departamento de Física Teórica, Universidad de Zaragoza, 50009 Zaragoza, Spain \\ e-mail: membrado@unizar.es \\ 2 BIFI, Instituto de Biofísica y Física de Sistemas Complejos, Universidad de Zaragoza, 50009 Zaragoza, Spain \\ e-mail: amalio@unizar.es
}

Received 11 July 2012 / Accepted 29 December 2012

\begin{abstract}
We calculate the range of possible masses for dark spheres of bound fully degenerate fermions as a function of the fermion mass. The cosmological constant is included in our calculations. We deduce that the minimum fermion mass that is able to give rise to degenerate fermion clusters is $\sim 0.02 g^{-1 / 4} \mathrm{eV}$, where $g$ is the spin degeneracy parameter. We show that degenerate fermions of $m c^{2} \approx$ $(15-30) g^{-1 / 4} \mathrm{eV}$ can build bound degenerate dark haloes that could reproduce the values of the rotation velocities of galaxies. The masses and radii derived for these degenerate dark haloes of typical galaxies, without considering any cosmological information, agree with the Jeans masses and radii of a cosmological background of $\sim(20-30) g^{-1 / 4} \mathrm{eV}$ degenerate fermions at redshift $z \approx 50$. However, degenerate fermion objects of $10^{15} M_{\odot}$ composed of these particles are too small to constitute the dark halo of galaxy clusters. We also derive degeneracy conditions for hot and cold dark matter fermions.
\end{abstract}

Key words. dark matter - dark energy

\section{Introduction}

It is accepted that most matter in the universe is dark. This belief comes from the fact that more mass than visible matter is required to explain the observed structure. At the end of the 1980s, it was suggested that baryonic dark matter could also be clumped in dark clusters (for a review, see for example, Carr 1994). The search for baryonic dark objects started at the beginning of the 1990s (see, for example, Moscoso 1993; Kerins \& Carr 1994; Wasserman \& Salpeter 1994). However, baryonic dark matter does not seem to be sufficient to describe the rotation curves observed in galaxies (see, for example, Caldwell 1995). Moreover, baryon-dominated models are ruled out by the observations of anisotropies of the cosmic microwave background (CMB; see, for example: Bond 1988; Strukov et al. 1987). Indeed, cosmological evidence favors non-baryonic dark matter (e.g., weakly interacting massive particles; see, for example, Trimble 1987), which reproduces rotational curves. The most natural non-baryonic dark matter candidate is the massive neutrino, but there are other alternatives such as the axion and various supersymmetric particles. The main argument in favor of non-baryonic dark matter comes from the primordial or big bang nucleosynthesis (BBN): it estimates a baryonic energy density much lower than present estimates of the energy density of matter. In addition, dominant non-baryonic dark matter models are consistent with other cosmological observations (the angular power spectrum of $\mathrm{CMB}$, the large-scale structure of the universe (LSS), lensing clusters), which also impose constraints on them (see, for example, Peacock 1999). In this work we will deal with fully degenerate fermionic dark matter. Semidegenerate massive neutrinos, with different degrees of degeneracy, have been extensively addressed; see, for example, Freese et al. (1983); Kang \& Steigman (1992); Lesgourgues \& Pastor (1999); Lesgourgues \& Liddle (2001); Dolgov et al. (2002b); and Dolgov (2002a).
The evolution of the non-baryonic dark matter distribution is collisionless. According to the Liouville theorem, the phasespace density of a collisionless system is preserved in its evolution. Therefore, the maximum phase-space density is conserved. Tremaine \& Gunn (1979) applied this result to leptons. They used the condition that the maximum phase-space density must not increase and that the energy density of the cosmological background of leptons must be lower than that of matter. They assumed that initially the distribution function is that of FermiDirac, very far from degeneracy, i.e., neglecting the chemical potential, and they took a Maxwellian velocity dispersion for the final relaxed cluster. Tremaine and Gunn concluded that massive galactic halos cannot be composed of stable neutral leptons of mass $\leq 1 \mathrm{MeV}$, which are relativistic when they decouple from radiation. Assuming a typical isothermal massive galactic halo, the phase-space constraint for neutrinos is $m c^{2}>33 \mathrm{eV}$ (see, for example, Peacock 1999), close to the result required for a critical-density universe. Thus, non-degenerate isothermal neutrinos cannot be the components of these dark haloes. However, neutrinos could constitute dark haloes in clusters of galaxies; for them, the constraint gives $m c^{2}>1.5 \mathrm{eV}$. Leptons with masses $>1 \mathrm{MeV}$ are non-relativistic at the decoupling, and they would not be ruled out by the phase-space density arguments.

In any case, the Tremaine and Gunn conclusion cannot be claimed for degenerate fermions such as those treated here. It must be taken into account that in a fully degenerate state, all quantum phase-space cells, from momentum 0 up to the Fermi momentum, are occupied. Therefore, the maximum phase-space density of fully degenerate fermions in bound clusters is equal to $g / h^{3}$, where $g$ is the spin degeneracy factor. Because in the evolution the maximum phase-space density cannot increase, the maximum phase-space density of the cosmological background of such fermions should also have been $g / h^{3}$. Hence, no constraint such as that derived by Tremaine and Gunn can be derived for the mass of the fermion. Nevertheless, in this work we look 
for restrictions on the mass of degenerate fermions by imposing the degeneracy condition on fermions that are relativistic when they decouple and on fermions that are non-relativistic at the decoupling.

There is strong evidence that the expansion of our flat universe (Efstathiou et al. 1999) is speeding up (see, for example: Perlmutter et al. 1997, 1999; Schmidt et al. 1998), which cannot be explained only in terms of matter and radiation. Thus, it is necessary to assume that another component is involved. This new component has been named "dark energy", and its pressure (from a hydrodynamical point of view) must be a negative function of its density. The first models for dark energy were hydrodynamical (Steinhardt 1996; Turner \& White 1997), but dynamical scalar field models were soon proposed (see, for example, Coble et al. 1997; Caldwell et al. 1998). When the dark energy density is assumed to be a constant and pressure is taken as minus density, a cosmological constant model is considered.

Pacheco et al. (1986) found that a cosmological constant imposes a minimum mass for the existence of Newtonian selfgravitating degenerate fermion systems. Though bound states were allowed by the repulsive effect of the cosmological constant, clusters could also suffer gravitational instability due to an excessive mass (see, for example, Shapiro \& Teukolski 1983). In this work, we study both the lower bound for the cluster mass imposed by the repulsive effect of the cosmological constant and the upper bound imposed by the gravitational instability.

Finally, we calculate the Jeans mass and Jeans length of a cosmological background of Newtonian degenerate fermions as a function of their redshift of formation.

The paper is organized as follows. In Sect. 2, Einstein equations are written for a fluid composed of a pure degenerate Fermi gas where the cosmological constant is present; the boundary conditions to solve these equations are also shown. In Sect. 3, we assume a weak field approximation to deal with Newtonian clusters. Section 4 shows the process we have followed to impose the stability conditions that limit the existence of these clusters. Numerical results are presented in Sect. 5. Section 6 deals with the degeneracy of a cosmological background of fermions as a function of the fermion mass. The Jeans mass for Newtonian degenerate fermions is calculated in Sect. 7. Finally, Sect. 8 contains our conclusions.

\section{Einstein equations}

We consider a static spherically symmetric cluster whose components are perfect fluids fulfilling different equations of state. The energy-stress-momentum tensor $T^{\mu \nu}$ is then given by (see, for example, Glendenning 1997)

$T^{\mu \nu}=-P g^{\mu \nu}+(P+\rho) U^{\mu} U^{v}$.

In Eq. (1), $P$ and $\rho$ are the pressure and the energy density of the fluid; $g_{\mu \nu}$ is the metric which, using polar coordinates $\left(x^{0}=c t\right.$, $\left.x^{1}=r, x^{2}=\theta, x^{3}=\phi\right)$, is given by

$g_{00}=B(r), \quad g_{11}=-A(r), \quad g_{22}=-r^{2}, \quad g_{33}=-r^{2} \sin ^{2} \theta$

$\left(\mathrm{d} s^{2}=B(r)(c \mathrm{~d} t)^{2}-A(r)(\mathrm{d} r)^{2}-r^{2}\left[(\mathrm{~d} \theta)^{2}+\sin ^{2}(\mathrm{~d} \phi)^{2}\right]\right.$ is therefore the line element $) ; U^{0}=\left(\mathrm{d} x^{0} / \mathrm{d} s\right)=B^{-1 / 2}$ and $\boldsymbol{U}=(\mathrm{d} \boldsymbol{x} / \mathrm{d} s)=$ $U^{0}(\boldsymbol{v} / c)=0$ are the components of the four-velocity tensor (they come from assuming a static cluster, so $\boldsymbol{v}=(\mathrm{d} \boldsymbol{x} / \mathrm{d} t)=0$, and from $\left.U^{\mu} U_{\mu}=1\right)$. $B(r), A(r), \rho(r)$ and $P(r)$ must be solutions of Einstein equations.

We consider a cluster composed of degenerate fermions of mass $m$ in a cosmological background of dark energy treated by the cosmological constant model. Then, the total energy density and pressure are:

$\rho=\rho_{\mathrm{F}}+\rho_{\Lambda}$,
$P=P_{\mathrm{F}}+P_{\Lambda}$,

where $\mathrm{F}$ and $\Lambda$ represent fermions and dark energy, respectively.

In a degenerate state, fermions have the lowest energy without violating the Pauli principle. In this state, all quantum phasespace cells, from zero momentum up to Fermi momentum $p_{\mathrm{F}}(r)$ (maximum momentum that a fermion can have at a distance $r$ from the cluster center), are occupied by $g$ fermions, where $g$ is the degeneracy per kinematic state. Hence, the distribution function, $f(p)$, called Fermi distribution function (see, for example, Kippenhahn \& Weigert 1994), is

$f(p)= \begin{cases}g / h^{3}, & 0 \leq p \leq p_{\mathrm{F}} \\ 0, & p_{\mathrm{F}}<p\end{cases}$

Thus, the number of fermions per unit volume whose absolute value of momentum lies between $p$ and $p+\mathrm{d} p$ is $4 \pi f(p) p^{2} \mathrm{~d} p$. Eq. (5) can be compared with the Fermi-Dirac distribution function (see Landau \& Lifshitz 2002),

$f_{\mathrm{FD}}(E)=\frac{g}{h^{3}}\left[\exp \left(\frac{E}{K_{\mathrm{B}} T}-\frac{\mu}{K_{\mathrm{B}} T}\right)+1\right]^{-1}$.

In Eq. (6), $\mu$ and $T$ are the chemical potential and the temperature of fermions, and $E$ is the kinetic energy related with the momentum, $p$, by

$E=\left[(p c)^{2}+\left(m c^{2}\right)^{2}\right]^{1 / 2}-m c^{2}$.

(Equation (6) can also be expressed as $f_{\mathrm{FD}}=\left(g / h^{3}\right)$ $\left[\exp \left(U / K_{\mathrm{B}} T-\mu^{*} / K_{\mathrm{B}} T\right)+1\right]^{-1}$, where $U=E+m c^{2}$ is the total energy and $\mu^{*}=\mu+m c^{2}$; see, for example, Raychaudhuri et al. 1992). As can be seen, when $\mu \gg K_{\mathrm{B}} T$, Eq. (6) coincides with Eq. (5) expressed as a function of the kinetic energy, $E$,

$f(E)= \begin{cases}g / h^{3}, & 0 \leq E \leq E_{\mathrm{F}}=\mu \\ 0, & E>E_{\mathrm{F}}=\mu .\end{cases}$

In Eq. (8), $E_{\mathrm{F}}$ is the Fermi kinetic energy, so

$E_{\mathrm{F}}=\left[\left(p_{\mathrm{F}} c\right)^{2}+\left(m c^{2}\right)^{2}\right]^{1 / 2}-m c^{2}$

The number density of particles, $n_{\mathrm{F}}$, is then

$n_{\mathrm{F}}=4 \pi \int_{0}^{\infty} f(p) p^{2} \mathrm{~d} p=\frac{4 \pi}{3} \frac{g}{h^{3}} p_{\mathrm{F}}^{3} ;$

and as a function of the dimensionless Fermi momentum, $x=p_{\mathrm{F}} / m c$,

$n_{\mathrm{F}}=g \frac{m^{3} c^{3} x^{3}}{6 \pi^{2} \hbar^{3}}$

(the end of a gravitationally bound system at $r=R$ is then characterized by the fact that no cell is occupied, so $p_{\mathrm{F}}(R)=0$, and therefore $n_{\mathrm{F}}(R)=0$ ). Energy density, $\rho_{\mathrm{F}}$, and pressure, $P_{\mathrm{F}}$, of degenerate fermions are given by

$\rho_{\mathrm{F}}=4 \pi \int_{0}^{\infty} f(p) U(p) p^{2} \mathrm{~d} p=\frac{g m^{4} c^{5}}{2 \pi^{2} \hbar^{3}} u(x)$,
$P_{\mathrm{F}}=\frac{4 \pi}{3} \int_{0}^{\infty} f(p) p v(p) p^{2} \mathrm{~d} p=\frac{g m^{4} c^{5}}{2 \pi^{2} \hbar^{3}} v(x)$, 
where $U(p)=\left(p^{2} c^{2}+m^{2} c^{4}\right)^{1 / 2}$ and $v(p)=p c /\left(p^{2}+m^{2} c^{2}\right)^{1 / 2}$ are the total energy and velocity of a fermion with momentum $p$, and

$$
\begin{aligned}
& u(x)=\frac{1}{8}\left[x\left(1+x^{2}\right)^{1 / 2}\left(2 x^{2}+1\right)-\ln \left(x+\left(1+x^{2}\right)^{1 / 2}\right)\right] \\
& v(x)=\frac{1}{24}\left[x\left(1+x^{2}\right)^{1 / 2}\left(2 x^{2}-3\right)+3 \ln \left(x+\left(1+x^{2}\right)^{1 / 2}\right)\right]
\end{aligned}
$$

For dark energy, we assume a cosmological constant model. Because the metric tensor $g^{\mu \nu}$ fulfills $g_{; \nu}^{\mu \nu}=0$, we can write a modified set of field equations, $G^{\mu v}+\Lambda g^{\mu \nu}=-\left(8 \pi G / c^{4}\right) T^{\mu v}$ from the Einstein equations ( $G^{\mu \nu}$ being Einstein tensor) that are also consistent with the conservation laws, $T_{; v}^{\mu \nu}=0 . \Lambda$ is the cosmological constant and was introduced by Albert Einstein. Therefore, the existence of an energy-momentum tensor of the vacuum can be considered, $T_{\mathrm{vac}}^{\mu \nu}=\frac{\Lambda c^{4}}{8 \pi G} g^{\mu \nu}$. From a hydrodynamical point of view, $T_{\text {vac }}^{\mu \nu}$ will agree with Eq. (1) if we take

$\rho_{\Lambda}=\frac{c^{4}}{8 \pi G} \Lambda$

$P_{\Lambda}=-\frac{c^{4}}{8 \pi G} \Lambda$.

Defining $\Omega_{\Lambda_{0}}=\rho_{\Lambda} / \rho_{c 0}$, where $\rho_{c 0}=\left(3 c^{2} H_{0}^{2}\right) /(8 \pi G)$ is the critical energy density of the universe at present (see, for example, Padmanabhan, 1995) and $H_{0}$ is the Hubble function at present, $\Lambda=3 H_{0}^{2} \Omega_{\Lambda 0} / c^{2}$; i.e.

$\Lambda=1.25 \times 10^{-56} C_{\Lambda 0} \mathrm{~cm}^{-2}$,

where

$C_{\Lambda 0}=\left(\frac{H_{0}}{70 \mathrm{~km} \mathrm{~s}^{-1} \mathrm{Mpc}^{-1}}\right)^{2}\left(\frac{\Omega_{\Lambda 0}}{0.73}\right)$.

Values for $H_{0}$ and $\Omega_{\Lambda 0}$ can be taken from Spergel et al. (2003).

In the absence of other forces, except gravitation, the nontrivial components of the field equation, $R_{\mu \nu}=-\left(8 \pi G / c^{4}\right)\left[T_{\mu \nu}-\right.$ $(1 / 2) g_{\mu \nu} T_{\alpha}^{\alpha}$, read as (see, for example, Weinberg 1972)

$R_{r r}=\frac{B^{\prime \prime}}{2 B}-\frac{B^{\prime}}{4 B}\left(\frac{A^{\prime}}{A}+\frac{B^{\prime}}{B}\right)-\frac{A^{\prime}}{r A}=-\frac{4 \pi G A}{c^{4}}(\rho-P)$,

$R_{\theta \theta}=-1+\frac{r}{2 A}\left(-\frac{A^{\prime}}{A}+\frac{B^{\prime}}{B}\right)+\frac{1}{A}=-\frac{4 \pi G r^{2}}{c^{4}}(\rho-P)$,

$R_{t t}=-\frac{B^{\prime \prime}}{2 A}+\frac{B^{\prime}}{4 A}\left(\frac{A^{\prime}}{A}+\frac{B^{\prime}}{B}\right)-\frac{B^{\prime}}{r A}=-\frac{4 \pi G B}{c^{4}}(\rho+3 P)$,

where $R_{\mu \nu}$ is the Ricci tensor and $T_{\mu \nu}$ is that of Eq. (1), with $\rho$ and $P$ given by Eqs. (3) and (4). In Eqs. (20)-(22), a prime represents $\mathrm{d} / \mathrm{d} r$. A fourth non-independent equation to (20)-(22) comes from the conservation of the energy-momentum tensor, $T_{; \gamma}^{\mu \nu}=0 ; T_{, v}^{0 v}$ is identical to null, and $T_{, v}^{1 v}=0$ gives the equation of the hydrostatic equilibrium of the system, i.e.,

$$
\frac{B^{\prime}}{B}=-\frac{2 P^{\prime}}{P+\rho}
$$

And a fifth equation is also obtained from Eqs. (20)-(22), as follows:

$$
\frac{r^{2}}{2}\left(\frac{R_{r r}}{A}+\frac{R_{t t}}{B}\right)+R_{\theta \theta}=\left(\frac{r}{A}\right)^{\prime}-1=-\frac{8 \pi G}{c^{4}} \rho r^{2} .
$$

Now, let us define a length scale, $a$, given by

$r=a y$,

$a=\left(\frac{3 \pi}{4 g}\right)^{1 / 2} \frac{\hbar^{3 / 2}}{G^{1 / 2} c^{1 / 2} m^{2}}=1.20 \times 10^{2}\left(\frac{g^{1 / 4} m c^{2}}{1 \mathrm{eV}}\right)^{-2} \mathrm{kpc}$.

Inserting (25) and (26) in (20)-(24), and using Eqs. (3), (4), (12), (13), (16) and (17), we have

$\frac{\ddot{B}}{2 B}-\frac{\dot{B}}{4 B}\left(\frac{\dot{A}}{A}+\frac{\dot{B}}{B}\right)-\frac{\dot{A}}{y A}=-\frac{3}{2} A\left(u(x)-v(x)+\frac{2}{3} a^{2} \Lambda\right)$,

$-1+\frac{y}{2 A}\left(-\frac{\dot{A}}{A}+\frac{\dot{B}}{B}\right)+\frac{1}{A}=-\frac{3}{2} y^{2}\left(u(x)-v(x)+\frac{2}{3} a^{2} \Lambda\right)$,

$-\frac{\ddot{B}}{2 A}+\frac{\dot{B}}{4 A}\left(\frac{\dot{A}}{A}+\frac{\dot{B}}{B}\right)-\frac{\dot{B}}{y A}=-\frac{3}{2} B\left(u(x)+3 v(x)-\frac{2}{3} a^{2} \Lambda\right)$,

$\frac{\dot{B}}{B}=-2 \frac{\dot{x} x}{\left(1+x^{2}\right)}$,

$-\frac{\dot{A}}{A^{2}} y+\frac{1}{A}-1=-3\left(u(x)+\frac{1}{3} a^{2} \Lambda\right) y^{2}$.

In Eqs. (27)-(31), a dot means d/dy. The value of $a$ given in Eq. (26) was chosen so that the coefficient on the right hand term in Eqs. (27)-(29) is $3 / 2$.

From Eqs. (27)-(31) coupled differential equations for $x$ and $A$ can be obtained. Using $\dot{B}$ (from Eq. (30)), $\dot{A}$ (from Eq. (31)) and $\ddot{B}$ (from the differentiation of Eq. (30) with respect to $y$ ) in Eq. (27), and after some manipulations, we obtain

$\ddot{x}=\frac{\dot{x}^{2}\left(2 x^{2}-1\right)}{x\left(1+x^{2}\right)}+\frac{\dot{A}}{A}\left(\frac{\dot{x}}{2}-\frac{1+x^{2}}{y x}\right)+\frac{3}{2} A \frac{1+x^{2}}{x}\left[F(x)+\frac{2}{3} a^{2} \Lambda\right]$,

where

$F(x)=\frac{1}{12} x\left(1+x^{2}\right)^{1 / 2}\left(2 x^{2}+3\right)-\frac{1}{4} \ln \left(x+\left(1+x^{2}\right)^{1 / 2}\right)$.

The differentiation of Eq. (31) with respect to the dimensionless radius $y$ gives

$$
\begin{aligned}
\ddot{A}= & \frac{A}{y^{2}}(A-1)+\frac{\dot{A}}{y}(1-2 A)+3 A(2 \dot{A} y+A) \\
& \times\left[G(x)+\frac{1}{3} a^{2} \Lambda\right] 3 y A^{2} \dot{x} x^{2}\left(1+x^{2}\right)^{1 / 2},
\end{aligned}
$$

where

$G(x)=\frac{1}{8}\left[x\left(1+x^{2}\right)^{1 / 2}\left(2 x^{2}+1\right)-\ln \left(x+\left(1+x^{2}\right)^{1 / 2}\right)\right]$.

If the cosmological constant terms were not considered in Eqs. (32) and (34), we would have the well-known TolmanOppenheimer-Volkoff equations and universal solutions for $x$ and $A$, i.e., solutions that are $m$ independent. However, as cosmological constant terms $\left(a^{2} \Lambda\right)$ depend on the fermion mass, it will be necessary to solve Eqs. (32) and (34) for each $m$.

From Eqs. (32) and (34), the behavior of $x$ and $A$ for $y \rightarrow 0$ can be deduced. Up to second order in $y$, we obtain

$$
\begin{aligned}
& x(y \rightarrow 0)=x_{0}+\frac{1+x_{0}^{2}}{2 x_{0}}\left[-2 G\left(x_{0}\right)+\frac{3}{2} F\left(x_{0}\right)+\frac{1}{3} a^{2} \Lambda\right] y^{2}, \\
& A(y \rightarrow 0)=1+\left[G\left(x_{0}\right)+\frac{1}{3} a^{2} \Lambda\right] y^{2} .
\end{aligned}
$$


According to Eqs. (36) and (37), the coupled differential equations (32) and (34) must be integrated with the four boundary conditions:

$x(0)=x_{0}, \dot{x}(0)=0, A(0)=1, \dot{A}(0)=0$.

The mass of the clusters inside a distance $r=a y$ is

$M(r)=\frac{4 \pi}{\mathrm{c}^{2}} \int_{0}^{r} \rho_{\mathrm{F}} r_{1}^{2} \mathrm{~d} r_{1}=\frac{3 \mathrm{c}^{2} a}{2 G} \int_{0}^{y} u(x) y_{1}^{2} \mathrm{~d} y_{1}$.

It can also be calculated by integrating Eq. (31),

$\frac{\mathrm{d}}{\mathrm{d} y}\left(\frac{y}{A}\right)=1-3\left[u(x)+\frac{1}{3} a^{2} \Lambda\right]$

from 0 up to some $y$. Thus,

$M(y)=\frac{c^{2}}{2 G}$ ay $\left[1-\frac{1}{A(y)}-\frac{\Lambda a^{2} y^{2}}{3}\right]$.

When $A$ is close to unity, it is better to use Eq. (39) to calculate $M(y)$. The radius of the cluster will be the distance, $R_{\mathrm{c}}=a y_{\mathrm{c}}$, that, as has been said above, fulfills $x\left(y_{\mathrm{c}}\right)=0$; so, the cluster mass will be $M_{\mathrm{c}}=M\left(y_{\mathrm{c}}\right)$.

The number of fermions inside a sphere of dimensionless radius $y$ is determined by

$N(y)=\frac{4 \pi a^{3}}{c^{2}} \int_{0}^{y} n_{\mathrm{F}}\left(y_{1}\right) y_{1}^{2} \mathrm{~d} y_{1}$,

where $n_{\mathrm{F}}$ is given by Eq. (10).

From Eq. (41), $A(y)$ can be expressed as

$A(y)=\left[1-\frac{2 G M(y)}{c^{2} a y}-\frac{\Lambda a^{2} y^{2}}{3}\right]^{-1}$.

With respect to $B$, the integration of Eq. (30),

$\frac{\mathrm{d} \ln B}{\mathrm{~d} x}=\ln \left(1+x^{2}\right)$,

from a point $y$ up to $y_{\mathrm{c}}$ (i.e., from $B(y)$ up to $B\left(y_{\mathrm{c}}\right)$ and from $x(y)$ up to $x\left(y_{\mathrm{c}}\right)=0$ ) leads to

$B(y)=\frac{B\left(y_{\mathrm{c}}\right)}{1+x^{2}(y)}$.

$B\left(y_{\mathrm{c}}\right)$ can be calculated from Eqs. (27) and (29) for $y \geq y_{\mathrm{c}}$ where $u=v=0$. Adding the product of $B$ and Eq. (27) to the product of $A$ and Eq. (29), we have

$\frac{\dot{A}}{A}=-\frac{\dot{B}}{B}, y \geq y_{\mathrm{c}}$

i.e., $\mathrm{d}(\ln (A B)) / \mathrm{d} y=0$, and therefore,

$A\left(y \geq y_{\mathrm{c}}\right) B\left(y \geq y_{\mathrm{c}}\right)=\mathrm{constant}=A(y=\infty) B(y=\infty)$.

At $y=\infty$, the metric given by Eq. (2) has to be the Minkowski metric (that of special relativity), so $A(y=\infty)=B(y=\infty)=1$; therefore

$B\left(y_{\mathrm{c}}\right)=\frac{1}{A\left(y_{\mathrm{c}}\right)}$.

And from Eq. (43) evaluated at $y_{\mathrm{c}}$,

$B\left(y_{\mathrm{c}}\right)=1-\frac{2 G M_{\mathrm{c}}}{c^{2} a y_{\mathrm{c}}}-\frac{\Lambda a^{2} y_{\mathrm{c}}^{2}}{3}$.

\section{Weak field approximation}

When fermions are non-relativistic $(x \ll 1)$, the weak field approximation can be considered; i.e.,

$A=1+\gamma,|\gamma| \ll 1$,

$B=1+\beta,|\beta| \ll 1$.

So, taking into account Eqs. (43), (45) and (49), the leading terms in $\gamma$ and $\beta$ are

$\gamma(y)=\frac{2 G M(y)}{c^{2} a y}+\frac{\Lambda a^{2} y^{2}}{3}$,

$\beta(y)=-x^{2}(y)-\frac{2 G M_{\mathrm{c}}}{c^{2} a y_{\mathrm{c}}}-\frac{\Lambda a^{2} y_{\mathrm{c}}^{2}}{3}$.

Using Eqs. (50) and (51) in Eq. (29), and taking into account that

$u(x \ll 1)=\frac{x^{3}}{3}$,

$v(x \ll 1)=\frac{x^{5}}{15}$,

we have

$\ddot{\beta}+\frac{2}{y} \dot{\beta}=x^{3}-2 a^{2} \Lambda$.

Equation (56) is the classical potential equation of gravity with

$\beta=2 \phi / c^{2}$,

$\phi$ being the gravitational potential; i.e.,

$\ddot{\phi}+\frac{2 \dot{\phi}}{y}=\frac{c^{2} x^{3}}{2}-c^{2} a^{2} \Lambda$.

To derive a differential equation for $x$ from Eq. (58), we can replace $\beta$ by $\phi$ (see (57)) in Eq. (53), i.e.,

$\phi=-\frac{c^{2} x^{2}}{2}-\frac{G M_{\mathrm{c}}}{a y_{\mathrm{c}}}-\frac{c^{2} \Lambda a^{2} y_{\mathrm{c}}^{2}}{6}$

and insert this equation in (58). Thus, we have

$\ddot{x}=-\frac{\dot{x}^{2}}{x}-\frac{2 \dot{x}}{y}-\frac{x^{2}}{2}+\frac{a^{2} \Lambda}{x}$.

From Eq. (60), the behavior of $x$ in the neighborhood of the cluster center, up to second order in $y$, is given by

$x(y \rightarrow 0)=x_{0}-\frac{1}{6}\left[\frac{x_{0}^{2}}{2}-\frac{a^{2} \Lambda}{x_{0}}\right] y^{2}$.

Evidently, Eq. (36) converts into Eq. (61) when $x_{0} \ll 1$ (taking into account that $\left.F\left(x_{0} \ll 1\right)=G\left(x_{0} \ll 1\right)=x_{0}^{3} / 3\right)$. Thus, from Eq. (61), Eq. (60) must be integrated with the boundary conditions

$x(0)=x_{0}, \dot{x}(0)=0$.

Using (54) in (39), (39) in (52), and (52) in (50), A can be expressed as

$A=1+\frac{1}{y} \int_{0}^{y} x^{3} y_{1}^{2} \mathrm{~d} y_{1}+\frac{a^{2} \Lambda}{3} y^{2}$ 
so,

$\dot{A}=-\frac{1}{y^{2}} \int_{0}^{y} x^{3} y_{1}^{2} \mathrm{~d} y_{1}+x^{3} y+\frac{2 a^{2} \Lambda}{3} y$.

The integral in Eqs. (63) and (64) can be expressed as a function of $x$ and $y$ as follows: integrating Eq. (56),

$\mathrm{d}\left(y^{2} \dot{\beta}\right)=\left(x^{3} y^{2}-2 \Lambda a^{2} y^{2}\right) \mathrm{d} y$,

from 0 up to some $y$, we have

$\dot{\beta}=\frac{1}{y^{2}} \int_{0}^{y} x^{3} y_{1}^{2} \mathrm{~d} y_{1}-\frac{2}{3} \Lambda a^{2} y$

and as from Eq. (53), $\dot{\beta}=-2 x \dot{x}$,

$\frac{1}{y} \int_{0}^{y} x^{3} y_{1}^{2} \mathrm{~d} y_{1}=-2 x \dot{x} y+\frac{2}{3} \Lambda a^{2} y^{2}$

Hence

$A=1-2 x \dot{x} y+a^{2} \Lambda y^{2}$,

$\dot{A}=2 x \dot{x}+x^{3} y$.

Instead of solving Eq. (60), we can also make the following changes of variable

$\phi=-\frac{G M_{\mathrm{c}}}{r} \chi-\frac{G M_{\mathrm{c}}}{a y_{\mathrm{c}}}-\frac{c^{2} \Lambda a^{2} y_{\mathrm{c}}^{2}}{6}$,

$r=b z$

Equating Eqs. (59) and (70), and using (71), we obtain that

$\left(\frac{2 G M_{\mathrm{c}}}{b c^{2}}\right) \frac{\chi}{z}=x^{2}$.

And using Eqs. (70)-(72) and (25) in (58), we have

$\frac{\mathrm{d}^{2} \chi}{\mathrm{d} z^{2}}=-\left(\frac{2 G M_{\mathrm{c}}}{b c^{2}}\right)^{1 / 2}\left(\frac{b}{a}\right)^{2}\left[\frac{\chi^{3 / 2}}{z^{1 / 2}}-2 a^{2}\left(\frac{b c^{2}}{2 G M_{\mathrm{c}}}\right)^{3 / 2} \Lambda z\right]$.

Equation (73) depends on two length scales ( $a$, given by Eq. (26), and the free parameter $b)$, on the mass of the cluster $\left(M_{\mathrm{c}}\right)$, and on the cosmological constant $(\Lambda)$. However, by taking

$\frac{2 G M_{\mathrm{c}}}{b c^{2}}=\left(\frac{a}{b}\right)^{4}$

we can make Eq. (73) depend on just one parameter. Thus, Eq. (73) reads

$\frac{\mathrm{d}^{2} \chi}{\mathrm{d} z^{2}}=-\frac{\chi^{3 / 2}}{z^{1 / 2}}+\alpha z$

where

$\alpha=\frac{2 b^{6}}{a^{4}} \Lambda$

Finally, using the value of $a$, given in Eq. (26), in Eqs. (74) and (76), $b$ and $\alpha$ are

$b=\left(\frac{9 \pi^{2}}{32 g^{2}}\right)^{1 / 3} \frac{\hbar^{2}}{G m^{8 / 3} M_{\mathrm{c}}^{1 / 3}}$,

$\alpha=\frac{9 \pi^{2}}{32 g^{2}} \frac{\hbar^{6} c^{2} \Lambda}{G^{4} m^{8} M_{\mathrm{c}}^{2}}$; i.e.,

$b=9.46\left(\frac{g^{1 / 4} m c^{2}}{20 \mathrm{eV}}\right)^{-8 / 3}\left(\frac{M_{\mathrm{c}}}{10^{11} M_{\odot}}\right)^{-1 / 3} \mathrm{kpc}$,

$\alpha=2.10 \times 10^{-5}\left(\frac{g^{1 / 4} m c^{2}}{20 \mathrm{eV}}\right)^{-8}\left(\frac{M_{\mathrm{c}}}{10^{11} M_{\odot}}\right)^{-2} C_{\Lambda 0}$.

In the neighborhood of the cluster center, the behavior of $\chi$ up to second order in $z$ is

$\chi(z \rightarrow 0)=\chi_{1} z-\frac{1}{6}\left[\chi_{1}^{3 / 2}-\alpha\right] z^{3}$.

Comparing (61) and (81), and taking into account (72) and (74), it can be seen that

$x_{0}=\left(\frac{a}{b}\right)^{2} \chi_{1}^{1 / 2}$.

Equation (75) has to be integrated for each value of $\alpha$, imposing the boundary conditions

$\chi(z=0)=0, \frac{\mathrm{d} \chi}{\mathrm{d} z}(z=0)=\chi_{1}$,

which derives from Eq. (81). For each $\alpha, \chi_{1}$ must be that which causes the mass condition to be fulfilled; i.e.,

$M_{\mathrm{c}}=4 \pi \int_{0}^{R_{\mathrm{c}}} m n_{\mathrm{F}} r^{2} \mathrm{~d} r$

where $R_{\mathrm{c}}=b z_{\mathrm{c}}$ with $\chi\left(z_{\mathrm{c}}\right)=0$. So using Eqs. (11), (72) and (79) in Eq. (84),

$1=\int_{0}^{z_{\mathrm{c}}} \chi^{3 / 2} z^{1 / 2} \mathrm{~d} z$

must be fulfilled.

Finally, when the cosmological constant is neglected, $\chi_{1}=$ 0.264 and $z_{\mathrm{c}}=5.10$, so the cluster radius is given by

$R_{\mathrm{c}}=48.1\left(\frac{g^{1 / 4} m c^{2}}{20 \mathrm{eV}}\right)^{-8 / 3}\left(\frac{M_{\mathrm{c}}}{10^{11} M_{\odot}}\right)^{-1 / 3} \mathrm{kpc}$.

\section{Minimum and maximum mass allowed for degenerate fermion clusters}

As pointed out in Sect. 2, because the $\Lambda$ terms in Eqs. (32) and (34) depend on fermion mass, it is necessary to solve these equations for each $m$. Thus, the discussion presented in the following paragraph must be made for each fermion mass.

The repulsive effect of the cosmological constant is already present in the neighborhood of the cluster center, as can be seen from Eqs. (36) and (37). According to Eq. (36), models with $x_{0}<x_{0}^{*}, x_{0}^{*}$ fulfilling

$-2 G\left(x_{0}^{*}\right)+\frac{3}{2} F\left(x_{0}^{*}\right)+\frac{1}{3} a^{2} \Lambda=0$,

show $\mathrm{d} x /\left.\mathrm{d} y\right|_{y=0}>0$ and are therefore unstable (see, for example, Jeans 1919; Shapiro \& Teukolski 1983). As we take greater $x_{0}$ 's, with $x_{0}>x_{0}^{*}$, the instability condition $\mathrm{d} x / \mathrm{d} y>0$ appears at greater dimensionless radii $y$ 's (by solving Eqs. (32) and (34)). This will continue to happen until some $x_{0}=x_{0, m}$, for which the instability condition appears exactly at $y=y_{\mathrm{c}, m}$ with $x\left(y_{\mathrm{c}, m}\right)=0$. The mass inside the sphere of dimensionless radius $y_{\mathrm{c}, m}$, calculated from Eq. (39), is denoted by $M_{m}$. Clusters 


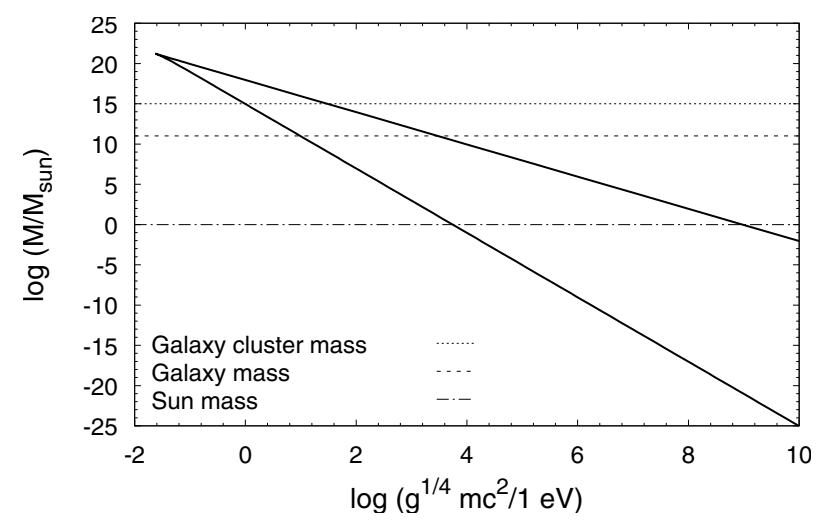

Fig. 1. Mass limits, $M_{M}$ (upper continuous line) and $M_{m}$ (lower continuous lines), versus fermion mass, $m$, for dark clusters of degenerate fermions. $C_{\Lambda 0}=1$ is assumed. For details see the text.

with $x_{0}>x_{0, m}$ do not fulfill $\mathrm{d} x / \mathrm{d} y>0$ at any $y \leq y_{\mathrm{c}}$, with $x\left(y_{\mathrm{c}}\right)=0$; therefore, $M_{m}$ is a lower limit for the mass of bound degenerate fermion clusters. The minimum number of fermions, $N_{m}$, that build a bound fermion cluster can then be calculated from Eq. (42) taking $y=y_{\mathrm{c}, m}$.

Pacheco et al. (1986) found, using Eq. (75) for nonrelativistic clusters, that $\alpha>\alpha^{*}=6.29 \times 10^{-3}$ does not allow bound states. Indeed, for $\alpha>\alpha^{*}, \mathrm{~d} x / \mathrm{d} z>0$ at some $z$, so such a cluster will be unstable. Therefore, for each fermion mass, $m$, $\alpha^{*}$ fixes the lower mass, $M_{m}$, for bound non-relativistic clusters. Taking $\alpha=\alpha^{*}$ in Eq. (75) and integrating that equation, the condition (85) is fulfilled for $\chi_{1}=0.180$ and $z_{\mathrm{c}}=7.71$. Thus, minimum mass, $M_{m}^{\mathrm{NR}}$ (from Eq. (80), taking $\alpha=\alpha^{*}=6.29 \times 10^{-3}$ ), minimum fermion number, $N_{m}^{\mathrm{NR}}=M_{m}^{\mathrm{NR}} / m$, and maximum radius, $R_{m}^{\mathrm{NR}}$ (using (79) in (71), with $z=z_{\mathrm{c}}=7.71$ and (88)), are

$$
\begin{aligned}
& M_{m}^{\mathrm{NR}}=9.24 \times 10^{14} C_{\Lambda 0}^{1 / 2}\left(\frac{g^{1 / 4} m c^{2}}{1 \mathrm{eV}}\right)^{-4} M_{\odot}, \\
& N_{m}^{\mathrm{NR}}=1.03 \times 10^{81} C_{\Lambda 0}^{1 / 2}\left(\frac{g^{1 / 4} m c^{2}}{1 \mathrm{eV}}\right)^{-5}, \\
& R_{m}^{\mathrm{NR}}=1.01 \times 10^{4} C_{\Lambda 0}^{-1 / 6}\left(\frac{g^{1 / 4} m c^{2}}{1 \mathrm{eV}}\right)^{-4 / 3} \mathrm{kpc} .
\end{aligned}
$$

Therefore,

$R_{m}^{\mathrm{NR}}=4.83 \times 10^{2} C_{\Lambda 0}^{-1 / 3}\left(\frac{M_{m}^{\mathrm{NR}}}{10^{11} M_{\odot}}\right)^{1 / 3} \mathrm{kpc}$.

Equation (60) could also be used to determine $M_{m}$; however, it would be necessary to calculate $x_{0, m}$ for each fermion mass, so the calculation time would be longer.

So far, we have dealt with lower bounds in the cluster mass. Now, we study upper bounds caused by the gravitational instability. There are $x_{0}>x_{0, m}$ models that are not stable. An upper limit for $x_{0}$, and therefore for the mass of clusters, comes from the condition

$\left.\frac{\mathrm{d} M_{\mathrm{c}}}{\mathrm{d} x_{0}}\right|_{x_{0, M}}=0$.

Equation (92) indicates that stability changes at $x_{0, M}$. Our $x_{0, m}<x_{0}$ models are stable for $x_{0}<x_{0, M}$ fulfilling $\mathrm{d} M_{\mathrm{c}} / \mathrm{d} x_{0}>0$, and unstable for $x_{0}>x_{0, M}$ fulfilling $\mathrm{d} M_{\mathrm{c}} / \mathrm{d} x_{0}<0$. Therefore, $M_{M}=M_{\mathrm{c}}\left(x_{0, M}\right)$ is the Oppenheimer-Volkoff limit for the mass of degenerate fermion clusters. When the repulsive effects of the

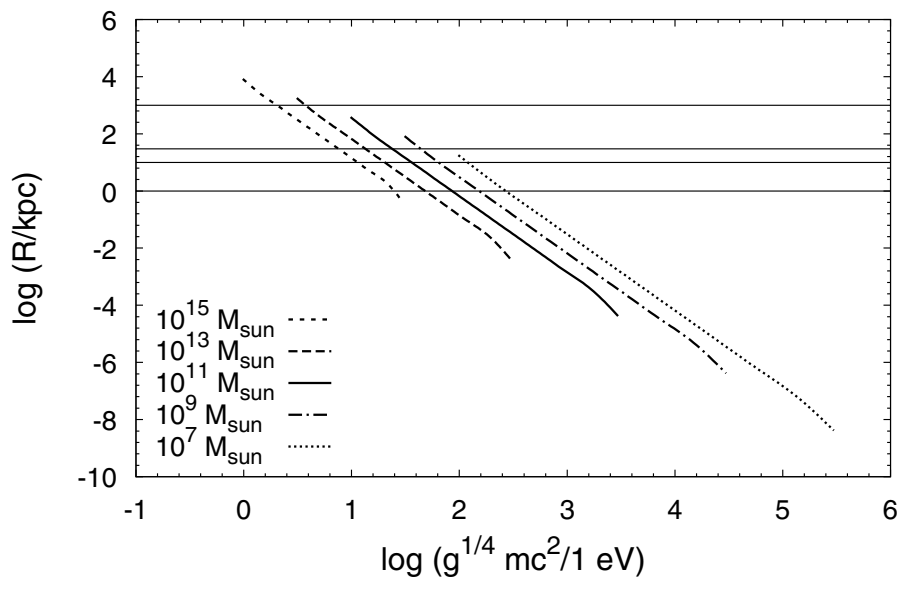

Fig. 2. Cluster radii, $R$, versus fermion mass, $m$, for several cluster masses, $M . C_{\Lambda 0}=1$ is assumed. Horizontal lines correspond to 1,10 , $30 \mathrm{kpc}$ and $1 \mathrm{Mpc}$. For details see the text.

cosmological constant are negligible, $M_{M}^{\mathrm{NCC}}, N_{M}^{\mathrm{NCC}}$ and $R_{M}^{\mathrm{NCC}}$ are given by

$M_{M}^{\mathrm{NCC}}=8.87 \times 10^{17}\left(\frac{g^{1 / 4} m c^{2}}{1 \mathrm{eV}}\right)^{-2} M_{\odot}$,
$N_{M}^{\mathrm{NCC}}=9.04 \times 10^{83}\left(\frac{g^{1 / 4} m c^{2}}{1 \mathrm{eV}}\right)^{-3}$,
$R_{M}^{\mathrm{NCC}}=3.72 \times 10^{2}\left(\frac{g^{1 / 4} m c^{2}}{1 \mathrm{eV}}\right)^{-2} \mathrm{kpc}$.

As a brief summary of this section, we emphasize that Eqs. (88)-(90) are non-relativistic results including repulsion effects, whilst Eqs. (93)-(95) are pure relativistic results that do not take repulsion into account.

A dimensional analysis of the lower bounds $M_{m}^{\mathrm{NR}}$ and upper bounds $R_{m}^{\mathrm{NR}}$ for fermions and bosons is described in Membrado $\&$ Pacheco (2012).

\section{Numerical results and discussion}

Numerically exact results for $M_{m}$ and $M_{M}$ versus fermion mass, $m c^{2}$, up to $10 g^{-1 / 4} \mathrm{GeV}$, are shown in the lower and upper curves of Fig. 1.

If relativistic effects are not taken into account in $M_{m}$ (from Eq. (88), $M_{m}^{\mathrm{NR}} \propto m^{-4}$ ) and if repulsive effects in $M_{M}$ are not considered (from Eq. (93), $M_{M}^{\mathrm{NCC}} \propto m^{-2}$ ), then, $M_{m}^{\mathrm{NR}}=M_{M}^{\mathrm{NCC}}=$ $8.51 \times 10^{20} C_{\Lambda 0}^{-1 / 2} M_{\odot}$ for $m c^{2}=3.22 \times 10^{-2} g^{-1 / 4} C_{\Lambda 0}^{1 / 4} \mathrm{eV}$. However, for this fermion mass, relativistic and repulsive effects can not be neglected. Our exact calculation, for $C_{\Lambda 0}=1$, gives $m_{*} c^{2}=2.38 \times 10^{-2} g^{-1 / 4} \mathrm{eV}, M_{*}=1.66 \times 10^{21} M_{\odot}$ (see the point in Fig. 1 where the two continuous lines intersect) and $R_{*}=1.26 \times 10^{3} \mathrm{Mpc} . m_{*}$ is, therefore, the minimim fermion mass that can build bound degenerate fermion clusters.

We can now compare values of $M_{m}$ obtained by neglecting relativistic effects, $M_{m}^{\mathrm{NR}}$ (see Eq. (88)), with those shown in Fig. 1. For fermion masses 1, 0.1, 0.04 and $0.02 g^{-1 / 4} \mathrm{eV}$, $100 \times\left|M_{m}^{\mathrm{NR}}-M_{m}\right| / M_{m}$ is about $0.1 \%, 0.6 \%, 10 \%$ and $72 \%$. Thus, we can say that relativistic effects on $M_{m}$ appear for $m \mathrm{c}^{2}<0.1 \mathrm{~g}^{-1 / 4} \mathrm{eV}$. With respect to $M_{M}$, the repulsive effects of the cosmological constant begin to be appreciable for $m c^{2}<0.1 \mathrm{~g}^{-1 / 4} \mathrm{eV}$; for $\mathrm{m}_{*} \mathrm{c}^{2}, M_{M}=M_{*}=1.66 \times 10^{21} M_{\odot}$ and $M_{M}^{\mathrm{NCC}}=1.56 \times 10^{21} M_{\odot}$, so $100 \times\left|M_{M}^{\mathrm{NCC}}-M_{M}\right| / M_{M} \approx 6 \%$. 
Numerical results for the radii of clusters, $R$, versus fermion mass, $m$, for several cluster masses, $M$, are shown in Fig. 2. In this figure, each line represents radii allowed for degenerate fermion clusters of the same mass, $M$. The maximum radius shown in each line corresponds to the limit imposed by the cosmological constant. The minimum radius in each line is the Oppenheimer-Volkoff limit.

We consider the mass of a typical galaxy dark halo $(G)$. This mass would be on the order of $\sim 10^{11} M_{\odot}$. According to Eq. (88) the minimum fermion mass that creates a degenerate cluster with $M_{m}^{\mathrm{NR}}=10^{11} M_{\odot}$ is $m_{\mathrm{m}, \mathrm{G}} c^{2}=9.80 \mathrm{~g}^{-1 / 4} C_{\Lambda 0}^{1 / 8} \mathrm{eV}$; from Eq. (90) we know that its radius is $R_{\mathrm{m}, \mathrm{G}}^{\mathrm{NR}}=482 C_{\Lambda 0}^{-1 / 3} \mathrm{kpc}$. Thus, the contributions to the rotational velocity would be about $v_{h}=\left(G M_{m}^{\mathrm{NR}} / R_{\mathrm{m}, \mathrm{G}}^{\mathrm{NR}}\right)^{1 / 2}=30 \mathrm{~km} \mathrm{~s}^{-1}$, too small to reproduce the rotational curve of a typical galaxy. The stability limit for $M_{M}^{\mathrm{NCC}}=10^{11} M_{\odot}$ is reached with $m_{\mathrm{M}, \mathrm{G}} c^{2}=2.97 \times 10^{3} \mathrm{~g}^{-1 / 4} \mathrm{eV}$ (see Eq. (93)); in that case, from Eq. (95), $R_{\mathrm{M}, \mathrm{G}}^{\mathrm{NCC}} \approx 4 \times 10^{-2}$ pc, so $v_{h}=10^{5} \mathrm{~km} \mathrm{~s}^{-1}$. Therefore, that degenerate cluster has too small a radius and too great a $v_{h}$ to be the dark halo of a typical galaxy. However, as we show below, there are fermion masses between $m_{\mathrm{m}, \mathrm{G}}$ and $m_{\mathrm{M}, \mathrm{G}}$ that could give rise to dark clusters whose $v_{h}$ could lead to the observed values of the rotational velocities of typical galaxies.

We now look at the mass of a dark halo of a typical galaxy cluster (GC), i.e., $\sim 10^{15} M_{\odot}$. If in Eq. (88) we take $M_{m}^{\mathrm{NR}}=$ $10^{15} M_{\odot}$, we conclude that the minimum fermion mass that can build a degenerate fermion object with such a mass is $m_{\mathrm{m}, \mathrm{GC}} c^{2}=$ $9.80 \times 10^{-1} g^{-1 / 4} C_{\Lambda 0}^{1 / 8} \mathrm{eV}$; and from Eq. (90), its radius is $R_{\mathrm{m}, \mathrm{GC}}^{\mathrm{NR}}=$ $10 C_{\Lambda 0}^{-1 / 3} \mathrm{Mpc}$. $v_{h}$ is then $\approx 650 \mathrm{~km} \mathrm{~s}^{-1}$, which is too small to reproduce the velocities of galaxies in clusters. It can also be seen that the maximum fermion mass that creates a $10^{15} M_{\odot}$ degenerate cluster is, from Eq. (93), $m_{\mathrm{M}, \mathrm{GC}} c^{2}=2.97 \times 10^{1} \mathrm{~g}^{-1 / 4} \mathrm{eV}$; in that case, from Eq. (95), $R_{\mathrm{M}, \mathrm{GC}}^{\mathrm{NCC}}=4.21 \times 10^{-1} \mathrm{kpc}$, so $v_{h} \approx$ $10^{5} \mathrm{~km} \mathrm{~s}^{-1}$. Therefore, the radius is too small and $v_{h}$ too high with respect to the size of a typical galaxy cluster and the velocities of their galaxies. We can also consider the case of $1 \mathrm{Mpc}$, which has about the visible size of a typical galaxy cluster. Figure 2 shows that a degenerate cluster of $10^{15} M_{\odot}$ and $1 \mathrm{Mpc}$ (so, $v_{h}=2 \times 10^{3} \mathrm{~km} \mathrm{~s}^{-1}$ ) can be built using a $2 \mathrm{~g}^{-1 / 4} \mathrm{eV}$ fermion (see Eq. (86)). However, this particle cannot build clusters with masses smaller than $5.8 \times 10^{13} M_{\odot}$ (see Eq. (88)), so it cannot be the component of the dark halos of galaxies.

It can be seen from Eq. (86) that fermions with masses between $15 g^{-1 / 4}$ and $30 g^{-1 / 4} \mathrm{eV}$ could create bound degenerate dark haloes that would contribute to the rotational velocity with values, $v_{h}=\left(G M_{\mathrm{c}} / R_{\mathrm{c}}\right)^{1 / 2}$, between 150 and $300 \mathrm{~km} \mathrm{~s}^{-1}$; these haloes would have radii between 15 and $50 \mathrm{kpc}$ and masses between $8 \times 10^{10} M_{\odot}$ and $7 \times 10^{11} M_{\odot}$. However, they could not compose the dark halo in a galaxy cluster, unless they were composed of dark galaxies. We consider some examples. For $20 g^{-1 / 4} \mathrm{eV}, M_{\mathrm{c}}=(2-6) \times 10^{11} M_{\odot}$ degenerate dark clusters show $R_{\mathrm{c}}=(38-27) \mathrm{kpc}$ and $v_{h}=(150-313) \mathrm{km} \mathrm{s}^{-1}$; and those with $M_{\mathrm{c}}=\left(10^{12}-10^{15}\right) M_{\odot}$ present $R_{\mathrm{c}}=(22-2.2) \mathrm{kpc}$ and $v_{h}=\left(4.4 \times 10^{2}-4.4 \times 10^{4}\right) \mathrm{km} \mathrm{s}^{-1}$, and therefore are incompatible with the observations of the largest galaxies and clusters of galaxies. For $25 \mathrm{~g}^{-1 / 4} \mathrm{eV}, M_{\mathrm{c}}=(1-4) \times 10^{11} M_{\odot}$ clusters are built with $R_{\mathrm{c}}=(26-17) \mathrm{kpc}$, showing $\mathrm{v}_{\mathrm{h}}=(129-318) \mathrm{km} \mathrm{s}^{-1}$; while for $M_{\mathrm{c}}=\left(10^{12}-10^{15}\right) M_{\odot}, R_{\mathrm{c}}=(12-1.3) \mathrm{kpc}$ and $\mathrm{v}_{\mathrm{h}}=$ $\left(5.9 \times 10^{2}-5.9 \times 10^{4}\right) \mathrm{km} \mathrm{s}^{-1}$, so they are again incompatible with observations.

For fermion masses smaller than $15 g^{-1 / 4} \mathrm{eV}$, only clusters with masses greater than $10^{12} M_{\odot}$ are able to give the kind of $v_{h}$ that is needed to reproduce the values of the rotational curves of typical galaxies; so, they cannot be the dark haloes of galaxies. The same conclusion is obtained for fermions with masses greater than $30 g^{-1 / 4} \mathrm{eV}$, but now because degenerate clusters show radii smaller than those of typical galaxies.

We now look at the problem of dwarf galaxies. Dwarf galaxies are the most abundant type of galaxy in the Universe. The largest dwarf irregulars have stellar masses of $\sim 10^{9} M_{\odot}$ and are rotationally supported, while dwarf spheroidal galaxies have stellar masses from $10^{7} M_{\odot}$ to below $10^{6} M_{\odot}$ with no rotational support (the lightest known is Segue 1 with a mass of $\sim 6 \times 10^{5} M_{\odot}$; see, for example, Simon et al. 2011).

High-resolution simulations of the standard $\Lambda \mathrm{CDM}$ (cosmological constant and cold dark matter) cosmology (see, for example: Klypin et al. 1999; Moore et al. 1999; Diemand et al. 2007; Springel et al. 2008) were applied to the Local Group; they showed a total number of dark matter subhaloes much greater than that of dwarf galaxies predicted using luminosity functions corrected by completeness and bias (Tollerud et al. 2008; Koposov et al. 2008) (one order of magnitud higher than the number of observed dwarf galaxies). It has been attempted to solve this problem found in $\Lambda \mathrm{CDM}$ simulations by suggesting that the supernova feedback could lead to the loss of baryonic components in haloes with masses smaller than a critical value, and that the effect of the photoionisation could prevent star formation in the smallest haloes (see, for example: Larson 1974; Dekel \& Silk 1986; Ferrara \& Tolstoy 2000; Efstathiou 1992; Somerville 2002; Hoeft et al. 2006; Simon \& Geha 2007). These mechanisms would make the dark matter mass-light ratio very high in haloes on the order of or smaller than $\sim 10^{9} M_{\odot}$ (Sawala et al. 2010).

Another problem found in simulations of dark halos using the standard $\Lambda \mathrm{CDM}$ cosmology comes from dark halos of $\sim 10{ }^{10} M_{\odot} . \Lambda \mathrm{CDM}$ simulations of $\sim(1-2) \times 10^{10} M_{\odot}$ dark halos show the formation of dwarf galaxies with stellar masses between $5 \times 10^{7} M_{\odot}$ and $2 \times 10^{8} M_{\odot}$ (Pelupessy et al. 2004; Stinson et al. 2007; Governato et al. 2010; Sawala et al. 2011). Guo et al. (2010) compared the abundance of dark haloes in the Millennium and Millennium-II Simulations to the observed abundances of galaxies as a function of the stellar mass obtained from SDSS DR-7 by Li \& White (2009). They found that the abundance of $\sim 10^{10} M_{\odot}$ dark haloes obtained from $\Lambda$ CDM simulations, and whose stellar mass is $\sim 5 \times 10^{7} M_{\odot}$, is about the abundance of observed galaxies with stellar mass of $\sim 10^{6} M_{\odot}$. Therefore, $\Lambda \mathrm{CDM}$ simulations predict galaxies with about fifty times more stellar mass than observed. In any case, if dark halos of $10^{10} M_{\odot}$ contain dwarf galaxies with stellar masses of $10^{8} M_{\odot}$, a $\Lambda$ CDM universe would predict about four times more of those galaxies than observed.

Thus, $\Lambda C D M$ simulations present serious problems for describing dwarf galaxies. In this respect, it should be said that warm dark matter models $(\Lambda \mathrm{WDM})$ predict about three times fewer dark haloes of $10^{10} M_{\odot}$ than $\Lambda$ CDM models for $m_{\mathrm{WDM}}=$ $1 \mathrm{keV}$ (Zavala et al. 2009).

We now look at our results for small degenerate dark haloes. We take as an example the case of fermions of $20 \mathrm{~g}^{-1 / 4} \mathrm{eV}$. According to Eq. (88), bound dark haloes with masses smaller than $6 \times 10^{9} C_{\Lambda 0}^{1 / 2} M_{\odot}$ cannot exist due to the repulsive effect of the cosmological constant. These small degenerate dark haloes would be very extensive (a degenerate dark halo of $M_{\mathrm{c}}=$ $10^{10} M_{\odot}$ would have a radius $R_{\mathrm{c}} \approx 100 \mathrm{kpc}$ ). However, these extensive degenerate dark haloes should be truncated by the tidal effects of larger neighboring galaxies (below we show as an example the case of the Large Magellanic Cloud). With respect to 
their contributions to the rotational velocities, $v_{h}=\left(G M_{\mathrm{c}} / R_{\mathrm{c}}\right)^{1 / 2}$, we see that they are small; thus, for a dark cluster of $10^{10} M_{\odot}$, $v_{h}(100 \mathrm{kpc}) \approx 20 \mathrm{~km} \mathrm{~s}^{-1}$. We may also wonder about the mass and velocity contribution at 5 and $10 \mathrm{kpc}$, which could be the size of dwarf galaxies contained in the dark haloes. For a $10^{10} M_{\odot}$ cluster, $M(5 \mathrm{kpc}) \approx 6.7 \times 10^{6} M_{\odot}$ and $v_{h}(5 \mathrm{kpc}) \approx 2.4 \mathrm{~km} \mathrm{~s}^{-1}$; $M(10 \mathrm{kpc}) \approx 5.4 \times 10^{7} M_{\odot}$ and $v_{h}(10 \mathrm{kpc}) \approx 4.8 \mathrm{~km} \mathrm{~s}^{-1}$.

We now consider the case of the Large Magellanic Cloud (LMC). This is a nearby barred spiral galaxy, a satellite of the Milky Way (MW). It is located at $D \approx 48.5 \mathrm{kpc}$ from the center of MW. Up to $8.9 \mathrm{kpc}$ its stellar disk mass is $\sim 2.7 \times 10^{9} M_{\odot}$ (de Vaucouleurs \& Freeman 1972; Bothum \& Thompson 1988; Zaritsky 1999; Bell \& de Jong 2001) and the neutral gas mass is $\sim 0.5 \times 10^{9} M_{\odot}$ (Kim et al. 1998); these masses contribute to the circular velocity of $39.3 \mathrm{~km} \mathrm{~s}^{-1}$. The circular velocity derived by Van der Marel et al. (2002) at $8.9 \mathrm{kpc}$ is $64.8 \pm 15.9 \mathrm{~km} \mathrm{~s}^{-1}$; consequently, a dark halo must exist in this galaxy.

The LMC suffers the tidal effects exerted by the MW. The gravitational interaction between LMC and MW is a very complex problem. But, for the sake of simplicity and merely to illustrate the problem, we have made very considerable simplifications. Therefore, the results described in the following must be considered as very rough estimates.

For the Milky Way we take, as an example, a degenerate dark halo composed of fermions of $23 g^{-1 / 4} \mathrm{eV}$ with a mass of $M_{\mathrm{MW}}=2.5 \times 10^{11} M_{\odot}$, and therefore a radius (see Eq. (86)) of $R_{\mathrm{MW}}=24.4 \mathrm{kpc}$. At the Sun position, $R_{\odot} \approx 8.5 \mathrm{kpc}$, the contribution to the rotational velocity is $v_{h} \approx 158 \mathrm{~km} \mathrm{~s}^{-1}$. Using the galactic bulge-disk model developed by Klypin et al. (2002), and taking for the bulge and disk masses, $M_{\mathrm{B}}=7 \times 10^{9} M_{\odot}$ and $M_{\mathrm{D}}=3.5 \times 10^{10} M_{\odot}$, the rotational velocity at $8.5 \mathrm{kpc}$ is $v_{\mathrm{r}}=\left(v_{\mathrm{d}}^{2}+v_{\mathrm{b}}^{2}+v_{h}^{2}\right)^{1 / 2} \approx 220 \mathrm{~km} \mathrm{~s}^{-1}, v_{\mathrm{d}}$ and $v_{\mathrm{b}}$ being the contributions of the bulge and the disk (we have taken $M_{\mathrm{D}} / M_{\mathrm{B}}=5$, as happens with the luminosity ratio $L_{\mathrm{D}} / L_{\mathrm{B}} \approx 5$ (see Kent et al. 1991); the $k$-band luminosity of the Galaxy measured by Kent et al. (1991) is $L_{K}=6.7 \times 10^{10} L_{\odot}$, and measured by Drimmel \& Spergel $\left.(2001), L_{K}=8.9 \times 10^{10} L_{\odot}\right)$. At $20 \mathrm{kpc}$, $v_{h} \approx 225 \mathrm{~km} \mathrm{~s}^{-1}$, which together with the contributions of the disk and bulge make $v_{\mathrm{r}} \approx 250 \mathrm{~km} \mathrm{~s}^{-1}$ (the rotation curve derived by Honma \& Sofue (1996) based on the geometry of the HI disk shows $\left.v_{\mathrm{r}} \approx 265 \pm 30 \mathrm{~km} \mathrm{~s}^{-1}\right)$.

For the LMC, we could propose a dark halo composed of $23 g^{-1 / 4} \mathrm{eV}$ degenerate fermions which, if isolated, would have $M_{\mathrm{c}}=8 \times 10^{10} M_{\odot}$ (according to Eq. (86), its radius would be $R_{\mathrm{c}}=35.7 \mathrm{kpc}$ ). However, this halo should be truncated by the gravitational effects of the MW. We can derive a rough estimate of a tidal radius, $r_{\mathrm{T}}$ and a mass of the degenerate dark halo, $M_{\mathrm{LMC}} \approx M\left(r_{\mathrm{T}}\right)$, as those that fulfill $G M_{\mathrm{MW}} / D^{2}+G M\left(r_{\mathrm{T}}\right) / r_{\mathrm{T}}^{2} \approx$ $G M_{\mathrm{MW}} /\left(D-r_{\mathrm{T}}\right)^{2}$. This equation is the balance of strengths per unit mass in the direction that joins the centers of the LMC and the MW. The first term on the left hand denotes the rotation of the LMC around the MW $\left(v_{\mathrm{LMC}}^{2} / D\right)$ and the other terms are the gravitational strength per unit mass due to the LMC and the MW at a distance $r_{\mathrm{T}}$ from the center of the LMC. For this calculation it is necessary to know $M(r)$. This can be derived by solving Eq. (75), neglecting the cosmological constant, and with the boundary conditions given by (83) and (85). Thus, we have obtained that $R_{\mathrm{LMC}}=r_{\mathrm{T}} \approx 14.5 \mathrm{kpc}$ and $M_{\mathrm{LMC}} \approx 2.3 \times 10^{10} M_{\odot}$. Assuming that the inner structure of the degenerate dark cluster is not changed by the truncation, the halo mass and the contribution to the circular velocity of LMC at $r=8.9 \mathrm{kpc}$ are $M(8.9 \mathrm{kpc}) \approx 6.5 \times 10^{9} M_{\odot}$ and $v_{h}(9 \mathrm{kpc}) \approx 56 \mathrm{~km} \mathrm{~s}^{-1}$. Accordingly, the total circular velocity would be $v_{\mathrm{r}}=69 \mathrm{~km} \mathrm{~s}^{-1}$, within the range given by Van der Marel et al. (2002). Smaller masses than $M_{\mathrm{c}}$ lead to smaller $r_{\mathrm{T}}, v_{h}$ and $v_{\mathrm{r}}$, while greater masses than $M_{\mathrm{c}}$ lead to greater $r_{\mathrm{T}}, v_{h}$ and $v_{\mathrm{r}}$.

With respect to dwarf spheroidal galaxies, we can consider the ultra-faint Milky Way satellite galaxy Segue 1 (Simon et al. 2011; Martinez et al. 2011). This is located at a distance of $23 \mathrm{kpc}$ from the MW center. The stars in this galaxy show a velocity dispersion $\sigma \approx 3.7 \mathrm{~km} \mathrm{~s}^{-1}$. This velocity dispersion could imply a dark halo mass of $M_{1 / 2} \approx 5.8 \times 10^{5} M_{\odot}$ within a sphere with a radius $r_{1 / 2} \approx 36 \mathrm{pc}$ that encloses half the stellar luminosity of the galaxy. As the $V$-band luminosity of Segue 1 is $240 L_{\odot}$, the $V$-band mass-to-light ratio within the half-light radius is $\approx 3400 M_{\odot} / L_{\odot}$. Assuming for the dark halo of Segue 1 a mass of $6 \times 10^{5} M_{\odot}$ and using the same formula as we used above to derive the tidal radius of $\mathrm{LMC}$, we obtain that the tidal radius of Segue 1 is $\approx 240 \mathrm{pc}$; this is much greater than $r_{1 / 2}$. This means that Segue 1 should not suffer tidal effects from the MW.

We assume that the dark halo of Segue 1 is composed of degenerate fermions like those proposed here to describe the dark halo of the MW. In that case, the condition that within a sphere of $36 \mathrm{pc}$ there is a mass of $5.8 \times 10^{5} M_{\odot}$ is fulfilled by a degenerate dark halo with a total mass of $\approx 3 \times 10^{12} M_{\odot}$ and a radius of $\approx 10 \mathrm{Kpc}$. This has no sense because the MW would suffer strong tidal effects. Therefore, the dark halo of Segue 1 cannot be composed of degenerate fermions like those proposed in this paper to describe the dark halo of typical galaxies. However, if the halo were composed of degenerate fermions of $\approx 1.3 \mathrm{keV}$, a cluster with total mass $M_{1 / 2}$ and radius $r_{1 / 2}$ could be reproduced (see Eq. (86)). The problem with this assumption is that, as can be seen from Eq. (125) shown below, such fermions are not degenerate; so Eq. (86) cannot be used. Therefore, such a non-degenerate fermion cluster with mass $M_{1 / 2}$ should have a radius greater than $r_{1 / 2}$.

Hence, it is apparent that to adequately model the dark halo of Segue 1 it is necessary to have more compact selfgravitating spheres than those provided by degenerate fermions. In this sense, spheres formed by fermions that are not completely degenerate are of no help because they are obviously less compact than their degenerate counterparts. Self-gravitating boson spheres, however, might be an answer. One kind of these objects is the self-gravitating collisionless Newtonian boson cluster in which all bosons occupy the lowest lying oneparticle Hartree orbital (Membrado et al. 1989a,b; Membrado $\&$ Aguerri 1996). For these condensates, the radius $R_{\mathrm{BC}}$, which covers $99 \%$ of the assembly, is $R_{\mathrm{BC}}=9.9\left(\hbar^{2} / G M_{\mathrm{BC}} m_{\mathrm{B}}^{2}\right)=$ $84.6\left(M_{\mathrm{BC}} / 10^{6} M_{\odot}\right)^{-1}\left(m_{\mathrm{B}} c^{2} / 10^{-20} \mathrm{eV}\right)^{-2} \mathrm{pc}$, where $M_{\mathrm{BC}}$ is the mass of the boson cluster and $m_{\mathrm{B}}$ the boson mass. An upper limit for the mass of the boson that could reproduce the dark halo of Segue 1 can be obtained assuming $M_{\mathrm{BC}}=M_{1 / 2}$ and $R_{\mathrm{BC}}=r_{1 / 2}$. Thus, $m_{\mathrm{B}} c^{2}<2 \times 10^{-20} \mathrm{eV}$. It should be said that invoking the uncertainly principle, it may be deduced that the linear momentum of each boson in the cluster is on the order of $p_{\mathrm{B}} \sim \hbar / R_{\mathrm{BC}}$ and hence that the de-Broglie wavelength (non-relativistic particles) for the boson is derived as $R_{\mathrm{BC}}$. Then, the phase-space condition for degeneracy in this condensate of bosons is trivially fulfilled: within a de-Broglie cell there are $M_{\mathrm{BC}} / m_{\mathrm{B}}$ particles.

Scalar fields fulfilling different potentials have been proposed to model dark matter (see, for example, Lee \& Koh 1996; Sahni \& Wang 2000; Matos \& Ureña-López 2000, 2001). The mass of this scalar dark matter, $m_{\mathrm{B}}$, ranges from $10^{-26} \mathrm{eV}$ up to $10^{-23} \mathrm{eV}$. The dark clusters composed of these fields show a minimal cutoff radius, greater than their Compton legth $\Lambda_{\mathrm{C}}=$ $\hbar / m_{\mathrm{B}} c$. Indeed, the enormous Compton length of these particles would prevent structure formation on small subgalactic 
scales. In this sense, collisionless boson spheres do not have this limitation.

Finally, we look at isolated dwarf galaxies of visible radii of about $10 \mathrm{Kpc}$. They show rotational velocities ranging between 50 and $150 \mathrm{~km} \mathrm{~s}^{-1}$ (see, for example, Kent 1987). Fermions with masses between $15 \mathrm{~g}^{-1 / 4}$ and $30 \mathrm{~g}^{-1 / 4} \mathrm{eV}$ could build more extensive degenerate structures that at $10 \mathrm{kpc}$ would show masses in the range $(0.6-5.2) \times 10^{10} M_{\odot}$. These would lead to rotational velocity contributions, $v_{h}$, similar to those shown by the visible dwarf galaxies. From the calculation of $M(r)$ for degenerate fermion clusters (by integrating Eq. (75) with the boundary conditions Eqs. (83) and (85)), it is deduced that $M(r) \propto r^{3}$ for approximately $r<0.4 R_{\mathrm{c}}$, so $v_{h}(r) \propto r$. Incidentally, this behavior of $v_{h}(r)$ is what is needed to describe the rotation velocity in some of those galaxies (see, for example, NGC 247, NGC 3109 , NGC 2259 from Kent 1987).

\section{Dependence on $m$ of the degeneracy of a cosmological background of fermions}

In this section we consider that the degenerate fermions decouple from radiation at an expansion parameter $a_{\mathrm{D}}$. For simplicity, we assume that the decoupling occurs instantaneously. Before the decoupling, the fermions are in equilibrium with the radiation, so their temperatures are equal, i.e., $T\left(a \leq a_{\mathrm{D}}\right)=$ $T_{\gamma}\left(a \leq a_{\mathrm{D}}\right)$. We assume that at $a_{\mathrm{D}}$, such fermions have a kinetic energy $E_{\mathrm{D}}$, a chemical potential $\mu_{\mathrm{D}}$ and a temperature $T_{\mathrm{D}}=T\left(a_{\mathrm{D}}\right)=T_{\gamma}\left(a_{\mathrm{D}}\right)$. Because we are considering degenerate fermions, $\mu_{\mathrm{D}} \gg K_{\mathrm{B}} T_{\mathrm{D}}$. We also assume that during $a_{\mathrm{NR}}<$ $a \leq a_{0}$ degenerate fermions are non-relativistic, and that their temperature at $a_{\mathrm{NR}}$ is $T_{\mathrm{NR}}=T\left(a_{\mathrm{NR}}\right)$.

We distinguish between fermions that are ultra-relativistic at $a_{\mathrm{D}}$ (hot and warm dark matter) and non-relativistic fermions at $a_{\mathrm{D}}$ (cold dark matter). The degeneracy condition $\mu_{\mathrm{D}} / K_{\mathrm{B}} T_{\mathrm{D}} \gg 1$ together with the condition $a_{\mathrm{NR}}>a_{\mathrm{D}}$ for the former and $a_{\mathrm{NR}}<a_{\mathrm{D}}$ for the latter must lead to restrictions on the mass of fermions. Obtaining these restrictions is the aim of this section.

Fermions that decouple when they are ultra-relativistic could be $e, \mu$ and $\tau$ neutrinos. Direct observational bounds on neutrino masses, found kinematically, are: $m_{v_{\mathrm{e}}}<10 \mathrm{eV}$ (Weinheimer et al. 1999; Lobashev et al. 1999; Particle Data Group 2000); $m_{v_{\tau}}<170 \mathrm{keV}$ (Assmagan et al. 1996); and $m_{v_{\tau}}<18 \mathrm{MeV}$ (Buskulic et al. 1995; Passalacqua 1997). In the absence of degeneracy, $e$-neutrinos decouple at $K_{\mathrm{B}} T_{\mathrm{D}}\left(v_{\mathrm{e}}\right) \approx 2.0 \mathrm{MeV}$, and $\mu$ and $\tau$ neutrinos at $K_{\mathrm{B}} T_{\mathrm{D}}\left(v_{\mu}\right) \approx K_{\mathrm{B}} T_{\mathrm{D}}\left(v_{\tau}\right) \approx 3.5 \mathrm{MeV}$ (see, for example, Freese et al. 1983). For semi-degenerate neutrinos, $T_{\mathrm{D}}$ is greater and depends on their degree of degeneration (see, for example, Kang \& Steigman, 1992). Heavy neutrinos could be an example of fermions that are non-relativistic when they decouple. For a review of neutrinos in cosmology, see, for example, Dolgov (2002a).

\subsection{Degenerate fermions that decouple when they are ultra-relativistic}

At $a_{\mathrm{D}}$, before the decoupling, the degenerate fermions, which are in equilibrium with the radiation, follow the Fermi distribution function given by Eq. (8)

$f_{\text {equi }}\left(E_{\mathrm{D}}, a_{\mathrm{D}}\right)= \begin{cases}g / h^{3}, & 0 \leq E_{\mathrm{D}} \leq \mu_{\mathrm{D}} \\ 0 & E_{\mathrm{D}}>\mu_{\mathrm{D}},\end{cases}$ where $E_{\mathrm{D}}$ and $\mu_{\mathrm{D}}$ are related with the momentum and the Fermi momentum at $a_{\mathrm{D}}, p_{\mathrm{D}}$ and $P_{\mathrm{FD}}$, by

$$
\begin{aligned}
& E_{\mathrm{D}}=\left[\left(p_{\mathrm{D}} c\right)^{2}+\left(m c^{2}\right)^{2}\right]^{1 / 2}-m c^{2} \\
& \mu_{\mathrm{D}}=\left[\left(p_{\mathrm{FD}} c\right)^{2}+\left(m c^{2}\right)^{2}\right]^{1 / 2}-m c^{2}
\end{aligned}
$$

If fermions are ultra-relativistic at $a_{\mathrm{D}}, p_{\mathrm{F}_{\mathrm{D}}} c / m c^{2} \gg 1$, only fermions with $p_{\mathrm{D}} c \gg m c^{2}$ contribute appreciably to physical quantities. Therefore, from Eqs. (97) and (98)

$E_{\mathrm{D}} \approx p_{\mathrm{D}} c$

$\mu_{\mathrm{D}} \approx p_{\mathrm{F}} c$,

and then Eq. (96) expressed as a function of the momentum, $p_{\mathrm{D}}$, is given by

$f_{\text {equi }}^{\mathrm{UR}}\left(p_{\mathrm{D}}, a_{\mathrm{D}}\right)= \begin{cases}g / h^{3}, & 0 \leq p_{\mathrm{D}} \leq \mu_{\mathrm{D}} / c \\ 0, & p_{\mathrm{D}}>\mu_{\mathrm{D}} / c .\end{cases}$

At $a_{\mathrm{D}}$, after the decoupling, the fermions travel along geodesics in space-time. During this free propagation, the distribution function of the fermions is conserved (the expansion of the universe causes the proper volume occupied by any number of these fermions to increase as $a^{3}$ while their volume in the momentum space is redshifted by $a^{-3}$ ). This allows us to obtain their distribution function after the decoupling, $f_{\mathrm{dec}}^{\mathrm{UR}}$. Thus, taking into account that as the universe expands, the fermion momentum, $p$, decreases as

$p(a)=p_{\mathrm{D}}\left(a_{\mathrm{D}} / a\right)$,

the conservation of the distribution function implies $f_{\mathrm{dec}}^{\mathrm{UR}}(p, a)=$ $f_{\text {equi }}^{\mathrm{UR}}\left(p a / a_{\mathrm{D}}, a_{\mathrm{D}}\right)$, so

$f_{\mathrm{dec}}^{\mathrm{UR}}(p, a)= \begin{cases}g / h^{3}, & 0 \leq p \leq\left(\mu_{\mathrm{D}} / c\right)\left(a_{\mathrm{D}} / a\right) \\ 0, & p>\left(\mu_{\mathrm{D}} / c\right)\left(a_{\mathrm{D}} / a\right) .\end{cases}$

Finally, as the fermion temperature, $T$, cools as

$T=T_{\mathrm{D}}\left(a_{\mathrm{D}} / a\right)$

even though the fermions are no longer in thermodynamical equilibrium with radiation (see, for example, Padmanabhan 1995), Eq. (103) reads as

$f_{\mathrm{dec}}^{\mathrm{UR}}(p)= \begin{cases}\left(g / h^{3}\right), & 0 \leq p \leq\left(\mu_{\mathrm{D}} / c\right)\left(T / T_{\mathrm{D}}\right) \\ 0, & p>\left(\mu_{\mathrm{D}} / c\right)\left(T / T_{\mathrm{D}}\right)\end{cases}$

Equation (105) tells us that the Fermi momentum, $p_{\mathrm{F}}$, is

$p_{\mathrm{F}}=\frac{\mu_{\mathrm{D}}}{c}\left(\frac{T}{T_{\mathrm{D}}}\right)$.

Hence, from Eq. (106), the fermions will be non-relativistic when $p_{\mathrm{F}} c / m c^{2}=\left(\mu_{\mathrm{D}} / m c^{2}\right)\left(T / T_{\mathrm{D}}\right) \ll 1$, and $T_{\mathrm{NR}}$ can then be estimated taking $p_{\mathrm{F}} c / m c^{2}=1$; thus,

$T_{\mathrm{NR}}=\frac{m c^{2}}{\mu_{\mathrm{D}}} T_{\mathrm{D}}$

Dividing now Eq. (107) by the fermion temperature at present, $T_{0}=T\left(a_{0}\right)$, and taking into account that $T / T_{0}=a_{0} / a$,

$\frac{a_{\mathrm{NR}}}{a_{0}}=\left(\frac{\mu_{\mathrm{D}}}{m c^{2}}\right) \frac{a_{\mathrm{D}}}{a_{0}}$. 
For $a>a_{\mathrm{NR}}$, degenerate fermions are non-relativistic. Then, their number density and energy density, $n_{\mathrm{F}}$ and $\rho_{\mathrm{F}}$, are given by

$$
\begin{aligned}
& n_{\mathrm{F}}=4 \pi \int_{0}^{\infty} f_{\mathrm{dec}}^{\mathrm{UR}}(p) p^{2} \mathrm{~d} p=\frac{g}{6 \pi^{2} \hbar^{3} c^{3}}\left[\mu_{\mathrm{D}}\left(\frac{T}{T_{\mathrm{D}}}\right)\right]^{3}, \\
& \rho_{\mathrm{F}}=m c^{2} n_{\mathrm{F}}=\frac{g m c^{2}}{6 \pi^{2} \hbar^{3} c^{3}}\left[\mu_{\mathrm{D}}\left(\frac{T}{T_{\mathrm{D}}}\right)\right]^{3},
\end{aligned}
$$

where the distribution function given by Eq. (105) has been used. Assuming a value for $\Omega_{\mathrm{F} 0}=\rho_{\mathrm{F} 0} / \rho_{c 0}$, the energy density at present will be

$\rho_{\mathrm{F} 0}=\frac{3 c^{2}}{8 \pi G} H_{0}^{2} \Omega_{\mathrm{F} 0}=2.23 \times 10^{-9} C_{\mathrm{F} 0} \mathrm{erg} \mathrm{cm}^{-3}$,

with

$C_{\mathrm{F} 0}=\left(\frac{H_{0}}{70 \mathrm{~km} \mathrm{Mpc}^{-1} \mathrm{~s}^{-1}}\right)^{2}\left(\frac{\Omega_{\mathrm{F} 0}}{0.27}\right) ;$

so, equating Eqs. (110) at $a_{0}$ and (111),

$\mu_{\mathrm{D}}\left(\frac{T_{0}}{T_{\mathrm{D}}}\right)=\left(\frac{9 \pi}{4 g}\right)^{1 / 3} \frac{\hbar c^{5 / 3}}{G^{1 / 2}\left(m c^{2}\right)^{1 / 3}}\left(H_{0}^{2} \Omega_{\mathrm{F} 0}\right)^{1 / 3}$.

Finally, using Eq. (113) in (107), we have

$\frac{T_{\mathrm{NR}}}{T_{0}}=\left(\frac{4 g}{9 \pi}\right)^{1 / 3} \frac{G^{1 / 3}\left(m c^{2}\right)^{4 / 3}}{\hbar c^{5 / 3}}\left(H_{0}^{2} \Omega_{\mathrm{F} 0}\right)^{-1 / 3} ;$

and, as $T / T_{0}=a_{0} / a$,

$\frac{a_{\mathrm{NR}}}{a_{0}}=\left(\frac{9 \pi}{4 g}\right)^{1 / 3} \frac{\hbar c^{5 / 3}}{G^{1 / 3}\left(m c^{2}\right)^{4 / 3}}\left(H_{0}^{2} \Omega_{\mathrm{F} 0}\right)^{1 / 3}$,

i.e.,

$\frac{a_{\mathrm{NR}}}{a_{0}}=8.61 \times 10^{-4} g^{-1 / 3}\left(\frac{m c^{2}}{1 \mathrm{eV}}\right)^{-4 / 3} C_{\mathrm{F} 0}^{1 / 3}$.

As an example, at $z=1000$ (a relatively short epoch after recombination when the growth of perturbations is fully given by linear theory and late enough for the decaying modes of perturbations to have decayed away), i.e., at $a / a_{0}=1 /(1+z)=9.99 \times 10^{-4}$, degenerate fermions are non-relativistic if

$m c^{2}>0.90 g^{-1 / 4} C_{\mathrm{F} 0}^{1 / 4} \mathrm{eV}$.

At present, the fermion temperature, $T_{0}$, differs from that of radiation, $T_{\gamma 0}$, due to different particle annihilation and decoupling occurring after $a_{\mathrm{D}}$. If only $\mathrm{e}^{+} \mathrm{e}^{-}$annihilate, $T_{0}=(4 / 11)^{1 / 3} T_{\gamma 0}$ (see, for example, Padmanabhan 1995). Thus, expressing the temperature of fermions at present, $T_{0}$, as

$T_{0}=1.96 F_{0}\left(\frac{T_{\gamma 0}}{2.75 \mathrm{~K}}\right)$,

with

$F_{0}=\left(\frac{T_{0} / T_{\gamma 0}}{(4 / 11)^{1 / 3}}\right)$

the expansion factor of the universe at the fermion decoupling, $a_{\mathrm{D}}$, can be expressed as

$\frac{a_{\mathrm{D}}}{a_{0}}=\frac{T_{0}}{T_{\mathrm{D}}}=1.69 \times 10^{-10}\left(\frac{K_{\mathrm{B}} T_{\mathrm{D}}}{1 \mathrm{MeV}}\right)^{-1} F_{0}\left(\frac{T_{\gamma 0}}{2.75 \mathrm{~K}}\right)$.
Thus, as degenerate fermions are relativistic when decoupling if $a_{\mathrm{D}} / a_{0}<a_{\mathrm{NR}} / a_{0}$, we deduce from Eqs. (116) and (120) that $m$ must fulfill

$m c^{2}<\frac{107}{g^{1 / 4}}\left(\frac{K_{\mathrm{B}} T_{\mathrm{D}}}{1 \mathrm{MeV}}\right)^{3 / 4} C_{\mathrm{F}_{0}}^{1 / 4} F_{0}^{-3 / 4}\left(\frac{T_{\gamma 0}}{2.75 \mathrm{~K}}\right)^{-3 / 4} \mathrm{keV}$

If the fermions were non-degenerate, we could estimate $a_{\mathrm{NR}} / a_{0}$ assuming $K_{\mathrm{B}} T_{\mathrm{NR}} \approx m c^{2} / 3$. Thus,

$\frac{a_{\mathrm{NR}}}{a_{0}}=\frac{T_{0}}{T_{\mathrm{NR}}}=\frac{3 K_{\mathrm{B}} T_{0}}{m c^{2}}=5.08 \times 10^{-4}\left(\frac{m c^{2}}{1 \mathrm{eV}}\right)^{-1} F_{0}\left(\frac{T_{\gamma 0}}{2.75 \mathrm{~K}}\right)$.

Therefore, non-degenerate fermions would be relativistic when decoupling $\left(a_{\mathrm{NR}} / a_{0}>a_{\mathrm{D}} / a_{0}\right)$ if

$m c^{2}<3.01\left(\frac{K_{\mathrm{B}} T_{\mathrm{D}}}{1 \mathrm{MeV}}\right) \mathrm{MeV}$.

The degeneracy parameter $\xi_{\mathrm{D}}=\mu_{\mathrm{D}} /\left(K_{\mathrm{B}} T_{\mathrm{D}}\right)$ can be estimated from Eqs. (113) and (118). Thus,

$\xi_{\mathrm{D}}=5.08 g^{-1 / 3}\left(\frac{m c^{2}}{1 \mathrm{eV}}\right)^{-1 / 3} C_{\mathrm{F} 0}^{1 / 3} F_{0}^{-1}\left(\frac{T_{\gamma 0}}{2.75 \mathrm{~K}}\right)^{-1}$.

Therefore, only fermions with mass $m$ fulfilling

$m c^{2} \ll 131 g^{-1} C_{\mathrm{F} 0} F_{0}^{-3}\left(\frac{T_{\gamma 0}}{2.75 \mathrm{~K}}\right)^{-3} \mathrm{eV}$

will be degenerate $\left(\xi_{\mathrm{D}} \gg 1\right)$. Evidently, the degeneracy condition (125) is more restrictive for $m$ than the relativistic condition at $a_{\mathrm{D}}$ given in (121).

Cosmological constraints on the neutrino degeneracy parameter began to be analyzed at the end of the past century (see, for example: Lesgourgue \& Pastor 1999; Pastor \& Lesgourgue 2000; Lesgourgue \& Liddle 2001; Orito et al. 2002; Dolgov et al. $2002 b)$. A relic neutrino degeneracy leads to a different amount of neutrinos and antineutrinos. Such a lepton asymmetry could have been imprinted on the cosmological data (Lesgourgue \& Pastor 1999, 2006). Studies about the effects of neutrino mass and neutrino degeneracy on the CMB, on the LSS, and on the $\mathrm{BBN}$ have been made in recent years, and their results have been compared with cosmological observations (such as data from the Wilkinson Microwave Anisotropy Probe (WMAP) on anisotropies of the CMB, from the BBN, and from the Sloan Digital Sky Survey (SDSS) on the dark matter power spectrum). Nevertheless, current constraints are still weak. WMAP could allow values of $\xi_{v}$ up to 4 (Shiraishi et al. 2009); however the BBN imposed bounds on $\left|\xi_{v}\right|$ smaller than 1.1 (see, for example: Serpico \& Raffet 2005; Lattanci et al. 2005; Hamann et al. 2008; Popa \& Vasile 2008; Shiraishi et al. 2009; Castorina et al. 2012).

We did not specify the type of degenerate fermion or its interactions; therefore, no comparison with cosmological data is made. Indeed, the aim of this subsection is simply to derive an upper limit for the mass of degenerate fermions that decouple when they are ultra-relativistic (as a function of their decoupling temperature and their temperature and energy density at present). We have also derived an expression for the degeneracy parameter (as a function of $m, T_{0}$ and $\rho_{\mathrm{F}_{0}}$ ). 


\subsection{Degenerate fermions that decouple when they are non-relativistic}

If degenerate fermions are non-relativistic at $a_{\mathrm{D}}, p_{\mathrm{FD}_{\mathrm{D}}} c / m c^{2} \ll 1$, and hence, $p_{\mathrm{D}} c / m c^{2} \ll 1$. Therefore, from Eq. (97), their kinetic energy, $E_{\mathrm{D}}$, is related to their momentum, $p_{\mathrm{D}}$, by

$E_{\mathrm{D}}=\frac{p_{\mathrm{D}}^{2}}{2 m}$

Therefore, using Eq. (126) in (96), the distribution function followed by these degenerate fermions at $a_{\mathrm{D}}$, before the decoupling, is

$f_{\text {equi }}^{\mathrm{NR}}\left(p_{\mathrm{D}}, a_{\mathrm{D}}\right)= \begin{cases}g / h^{3}, & 0 \leq p_{\mathrm{D}} \leq\left(2 m \mu_{\mathrm{D}}\right)^{1 / 2} \\ 0, & p_{\mathrm{D}}>\left(2 m \mu_{\mathrm{D}}\right)^{1 / 2}\end{cases}$

Thus, taking into account the conservation of the distribution function together with Eqs. (102) and (104), the distribution function after the decoupling, $f_{\mathrm{dec}}^{\mathrm{NR}}$, will be

$f_{\text {dec }}^{\mathrm{NR}}(p)= \begin{cases}g / h^{3}, & 0 \leq p \leq\left(2 m \mu_{\mathrm{D}}\right)^{1 / 2}\left(T / T_{\mathrm{D}}\right) \\ 0, & p>\left(2 m \mu_{\mathrm{D}}\right)^{1 / 2}\left(T / T_{\mathrm{D}}\right)\end{cases}$

Hence, the Fermi momentum, $p_{\mathrm{F}}$, is now

$p_{\mathrm{F}}=\left(2 m \mu_{\mathrm{D}}\right)^{1 / 2}\left(\frac{T}{T_{\mathrm{D}}}\right)$

According to Eq. (129), fermions are non-relativistic when $p_{\mathrm{F}} c / m c^{2}=\left(2 \mu_{\mathrm{D}} / m c^{2}\right)^{1 / 2}\left(T / T_{\mathrm{D}}\right) \ll 1$; so $T_{\mathrm{NR}}$, estimated taking $p_{\mathrm{F}} c / m c^{2}=1$, is

$T_{\mathrm{NR}}=\left(\frac{m c^{2}}{2 \mu_{\mathrm{D}}}\right)^{1 / 2} T_{\mathrm{D}}$

and therefore,

$\frac{a_{\mathrm{NR}}}{a_{0}}=\left(\frac{2 \mu_{\mathrm{D}}}{m c^{2}}\right)^{1 / 2} \frac{a_{\mathrm{D}}}{a_{0}}$.

Using Eq. (128) for non-relativistic degenerate fermions, their number density and energy density, $n_{\mathrm{F}}$ and $\rho_{\mathrm{F}}$, are given by

$n_{\mathrm{F}}=4 \pi \int_{0}^{\infty} f_{\mathrm{dec}}^{\mathrm{NR}}(p) p^{2} \mathrm{~d} p=\frac{2^{1 / 2} g\left(m c^{2}\right)^{3 / 2}}{3 \pi^{2} \hbar^{3} c^{3}}\left[\mu_{\mathrm{D}}\left(\frac{T}{T_{\mathrm{D}}}\right)^{2}\right]^{3 / 2}$

$\rho_{\mathrm{F}}=m c^{2} n_{\mathrm{F}}=\frac{2^{1 / 2} g\left(m c^{2}\right)^{5 / 2}}{3 \pi^{2} \hbar^{3} c^{3}}\left[\mu_{\mathrm{D}}\left(\frac{T}{T_{\mathrm{D}}}\right)^{2}\right]^{3 / 2}$.

Thus, equating (133) at $a_{0}$ with (111),

$\mu_{\mathrm{D}}\left(\frac{T_{0}}{T_{\mathrm{D}}}\right)^{2}=\left(\frac{81 \pi^{2}}{128 g^{2}}\right)^{1 / 3} \frac{\hbar^{2} c^{10 / 3}}{G^{2 / 3}\left(m c^{2}\right)^{5 / 3}}\left(H_{0}^{2} \Omega_{\mathrm{F} 0}\right)^{2 / 3}$.

Now, using Eq. (134) in (130), we have

$\frac{T_{\mathrm{NR}}}{T_{0}}=\left(\frac{4 g}{9 \pi}\right)^{1 / 3} \frac{G^{1 / 3}\left(m c^{2}\right)^{4 / 3}}{\hbar c^{5 / 3}}\left(H_{0}^{2} \Omega_{\mathrm{F} 0}\right)^{-1 / 3}$,

and, therefore,

$\frac{a_{\mathrm{NR}}}{a_{0}}=\left(\frac{9 \pi}{4 g}\right)^{1 / 3} \frac{\hbar c^{5 / 3}}{G^{1 / 2}\left(m c^{2}\right)^{4 / 3}}\left(H_{0}^{2} \Omega_{\mathrm{F} 0}\right)^{1 / 3}$, i.e.,

$\frac{a_{\mathrm{NR}}}{a_{0}}=8.61 \times 10^{-4} g^{-1 / 3}\left(\frac{m c^{2}}{1 \mathrm{eV}}\right)^{-4 / 3} C_{\mathrm{F} 0}^{1 / 3}$.

Equations (135)-(137) are the same equations as derived for degenerate fermions that are ultra-relativistic at $a_{\mathrm{D}}$ (see Eqs. (114)-(116)). Finally, taking into account that degenerate fermions are non-relativistic at $a_{\mathrm{D}}$ when $a_{\mathrm{D}} / a_{0}>a_{\mathrm{NR}} / a_{0}$, we have, from Eqs. (137) and (120), that $m$ must fulfill

$m c^{2}>\frac{107}{g^{1 / 4}}\left(\frac{K_{\mathrm{B}} T_{\mathrm{D}}}{1 \mathrm{MeV}}\right)^{3 / 4} C_{\mathrm{F} 0}^{1 / 4} F_{0}^{-3 / 4}\left(\frac{T_{\gamma 0}}{2.75 \mathrm{~K}}\right)^{-3 / 4} \mathrm{keV}$.

From Eqs. (134) and (118), the degenerate parameter, $\xi_{\mathrm{D}}$ is now,

$$
\begin{aligned}
\xi_{\mathrm{D}}= & 6.01 \times 10^{-2} g^{-2 / 3}\left(\frac{m c^{2}}{100 \mathrm{keV}}\right)^{-5 / 3}\left(\frac{K_{\mathrm{B}} T_{\mathrm{D}}}{1 \mathrm{MeV}}\right) \\
& \times C_{\mathrm{F} 0}^{2 / 3} F_{0}^{-2}\left(\frac{T_{\gamma 0}}{2.75 \mathrm{~K}}\right)^{-2} .
\end{aligned}
$$

So using (138) in (139),

$\xi_{\mathrm{D}}<\frac{5.36 \times 10^{-2}}{g^{2 / 3}}\left(\frac{K_{\mathrm{B}} T_{\mathrm{D}}}{1 \mathrm{MeV}}\right)^{-1 / 4} C_{\mathrm{F} 0}^{1 / 4} F_{0}^{-3 / 4}\left(\frac{T_{\gamma 0}}{2.75 \mathrm{~K}}\right)^{-3 / 4}$.

Therefore, according to Eqs. (140), fermions coming from a cosmological background that decouple when they are nonrelativistic cannot be completely degenerate.

\section{Jeans mass for Newtonian degenerate fermions}

The Jeans length for a background of Newtonian degenerate fermions can be derived by studying a perturbation solution from the unperturbed state, which is taken as spatially uniform in the neighborhood of the perturbation. The simplest procedure is to decompose the perturbation of a magnitude $\chi$ into a system of plane waves with wave vector $\boldsymbol{K}$ and instability growth $\omega$ (see, for example, Binney \& Tremaine 1987; in this paper we follow the work by Membrado \& Aguerri 1996); i.e. $\chi=$ $\chi_{u}+\chi_{p} \exp [\omega t+\mathrm{i} \boldsymbol{K} \cdot \boldsymbol{r}], \chi$ being, in this section, the energy density, $\rho$ (related with the density number, $n$, by $\rho=m c^{2} n$ ), velocity, $\boldsymbol{v}$, and gravitational potential, $\phi$. Magnitudes with subindex $u$ describe the unperturbed solution, while those with subindex $p$ describe small amplitudes of the perturbation. Inserting the perturbation and the unperturbed solution in the hydrodynamical equations

$$
\begin{aligned}
& \frac{1}{c^{2}} \frac{\partial \rho}{\partial t}+\frac{1}{c^{2}} \boldsymbol{\nabla} \cdot[\rho \boldsymbol{v}]=0 \\
& \frac{\rho}{c^{2}}\left[\frac{\partial \boldsymbol{v}}{\partial t}+\boldsymbol{v} \cdot \boldsymbol{\nabla} \boldsymbol{v}+\boldsymbol{\nabla} \phi\right]+\nabla P=0
\end{aligned}
$$

and in the Poisson equation

$\nabla^{2} \phi=\frac{4 \pi G \rho}{c^{2}}$

and neglecting second-order terms, we have

$$
\begin{aligned}
& \omega \frac{\rho_{p}}{c^{2}}+\mathrm{i} \boldsymbol{K} \cdot \boldsymbol{v}_{p} \frac{\rho_{u}}{c^{2}}=0, \\
& \omega \boldsymbol{v}_{p} \frac{\rho_{u}}{c^{2}}+\mathrm{i} \boldsymbol{K} \phi_{p} \frac{\rho_{u}}{c^{2}}+\left.\mathrm{i} \boldsymbol{K} \rho_{p} \frac{\mathrm{d} P}{\mathrm{~d} \rho}\right|_{u}=\mathbf{0}, \\
& -K^{2} \phi_{p}=4 \pi G \frac{\rho_{p}}{c^{2}} .
\end{aligned}
$$


Equations (144)-(146) are therefore the equation of continuity, the equation of motion, and the Poisson equation. In Eq. (145), $P$ is the pressure that fulfills an equation of state $P=P(\rho)$. To simplify, we assume that the unperturbed state is at rest $\left(\boldsymbol{v}_{u}=0\right)$.

We calculate the Jeans mass of a cosmological background of degenerate fermions at an expansion factor fulfilling $a_{\mathrm{NR}}<$ $a<a_{0}$, therefore we consider that the fermions are nonrelativistic.

For degenerate fermions that are ultra-relativistic at $a_{\mathrm{D}}$, their pressure, $P_{\mathrm{UR}}$, as a function of their energy density, $\rho_{\mathrm{UR}}$, can be calculated from

$P_{\mathrm{UR}}=\frac{4 \pi}{3} \int_{0}^{\infty} f_{\mathrm{dec}}^{\mathrm{UR}}(p) p v(p) p^{2} \mathrm{~d} p=\frac{g}{30 \pi^{2} \hbar^{3} c^{5} m}\left[\mu_{\mathrm{D}}\left(\frac{T}{T_{\mathrm{D}}}\right)\right]^{5}$,

where $f_{\mathrm{dec}}^{\mathrm{UR}}$ is given by (105) and $v=p / m$ is the fermion velocity. Thus, taking into account that $\rho_{\mathrm{UR}}$ is given by Eq. (110), the equation of state is

$P_{\mathrm{UR}}=\frac{6^{2 / 3} \pi^{4 / 3} \hbar^{2} c^{2}}{5 g^{2 / 3}\left(m c^{2}\right)^{8 / 3}} \rho_{\mathrm{UR}}^{5 / 3}$

The pressure, $P_{\mathrm{NR}}$, of degenerate fermions that are nonrelativistic when decoupling is

$P_{\mathrm{NR}}=\frac{4 \pi}{3} \int_{0}^{\infty} f_{\mathrm{dec}}^{\mathrm{NR}}(p) p v(p) p^{2} \mathrm{~d} p=\frac{2^{3 / 2} g m^{3 / 2}}{15 \pi^{2} \hbar^{3}}\left[\mu_{\mathrm{D}}\left(\frac{T}{T_{\mathrm{D}}}\right)^{2}\right]^{5 / 2}$,

where $f_{\text {dec }}^{\mathrm{NR}}$ is given by (128) and $v=p / m$. So using $\rho_{\mathrm{NR}}$ from Eq. (133) leads to the same equation of state as that shown in (148), i.e.,

$P_{\mathrm{NR}}=\frac{6^{2 / 3} \pi^{4 / 3} \hbar^{2} c^{2}}{5 g^{2 / 3}\left(m c^{2}\right)^{8 / 3}} \rho_{\mathrm{NR}}^{5 / 3}$.

Thus from Eq. (148) or (150)

$\left.\frac{\mathrm{d} P}{\mathrm{~d} \rho}\right|_{u}=\frac{6^{2 / 3} \pi^{4 / 3} \hbar^{2} c^{2}}{3 g^{2 / 3}\left(m c^{2}\right)^{8 / 3}} \rho_{u}^{2 / 3}$.

Equations (144)-(146) together with (151) have a non-trivial solution if

$\omega^{2}=4 \pi G \frac{\rho_{u}}{c^{2}}-K^{2} \frac{6^{2 / 3} \pi^{4 / 3} \hbar^{2} c^{4}}{3 g^{2 / 3}\left(m c^{2}\right)^{8 / 3}} \rho_{u}^{2 / 3}$.

Thus, there exists a critical value of $K_{\mathrm{J}}$ determined by the condition $\omega=0$. The Jeans length is then given by

$\lambda_{\mathrm{J}}=\frac{2 \pi}{K_{\mathrm{J}}}=\frac{2^{1 / 3} \pi^{7 / 6}}{3^{1 / 6} g^{1 / 3}} \frac{\hbar c^{3}}{G^{1 / 2}\left(m c^{2}\right)^{4 / 3} \rho_{u}^{1 / 6}}$,

and the Jeans mass, $M_{\mathrm{J}}=\left(4 \pi \rho_{u} / 3 c^{2}\right)\left(\lambda_{\mathrm{J}} / 2\right)^{3}$, is

$M_{\mathrm{J}}=\frac{\pi^{9 / 2}}{3^{3 / 2} g} \frac{\hbar^{3} c^{7} \rho_{u}^{1 / 2}}{G^{3 / 2}\left(m c^{2}\right)^{4}}$.

If $\lambda>\lambda_{\mathrm{J}} 3$, i.e. if $M>M_{J}$, then $\omega^{2}>0$ and a cluster can be created.

Equations (153) and (154) can be used to estimate the Jeans length and mass at different redshifts. For this purpose, we assume that $\rho_{u}$ is the background energy density of degenerate fermions at some $z$. Taking into account that we are dealing with non-relativistic matter, $\rho(z)=\rho(z=0)(1+z)^{3}$ (see, for example, Padmanabhan 1995), and using (111), we have

$\rho_{u} \equiv \rho_{\mathrm{F}}(z)=2.23 \times 10^{-9}(1+z)^{3} C_{\mathrm{F} 0} \operatorname{erg~\mathrm {cm}^{-3}}$.
Therefore,

$\lambda_{J}(z)=387(1+z)^{-1 / 2}\left(\frac{g^{1 / 4} m c^{2}}{20 \mathrm{eV}}\right)^{-4 / 3} C_{\mathrm{F} 0}^{-1 / 6} \mathrm{kpc}$,

$M_{J}(z)=1.11 \times 10^{9}(1+z)^{3 / 2}\left(\frac{g^{1 / 4} m c^{2}}{20 \mathrm{eV}}\right)^{-4} C_{\mathrm{F} 0}^{1 / 2} M_{\odot}$.

We now consider redshift $z=50$, when the non-linear collapse could have started (see for example, Padmanabhan 1995). At this redshift, the Jeans mass and radius of a cosmological background of $m c^{2} \approx 20-30 g^{-1 / 4} \mathrm{eV}$ degenerate fermions is $M_{\mathrm{J}} \approx(4-0.8) \times 10^{11} C_{\mathrm{F} 0}^{1 / 2} M_{\odot}$ and $R_{\mathrm{J}} \approx \lambda_{\mathrm{J}} / 2 \approx(27-16) C_{\mathrm{F} 0}^{-1 / 6} \mathrm{kpc}$ (see Eqs. (157) and (156)). These results could be consistent with a typical galaxy dark halo. In addition, these results based on cosmological background densities at $z=50$ are coincident with those shown in Sect. 5, where no cosmological information was used (only the relation among fermion mass and bound degenerate cluster mass and radius). For those fermion masses, a Jeans mass of $10^{15} M_{\odot}$ is reached at $z \approx(0.9-2.8) \times 10^{4} C_{\mathrm{F} 0}^{-1 / 3}$, when $R_{\mathrm{J}} \approx(2-0.7) \mathrm{kpc}$; therefore, dark halos of clusters of galaxies cannot be composed of such degenerate fermions. For $M_{J}=10^{10} M_{\odot}, z \approx(4-13) C_{\mathrm{F} 0}^{-1 / 3}$ and $R_{\mathrm{J}} \approx 58-31 \mathrm{kpc}$.

At redshift $z=50$, a background of degenerate fermions of $m c^{2} \approx 2.8 g^{-1 / 4} \mathrm{eV}$ shows $M_{\mathrm{J}} \approx 10^{15} C_{\mathrm{F} 0}^{1 / 2} M_{\odot}$ (according to Eq. (88), $2.8 g^{-1 / 4} \mathrm{eV}$ fermions cannot build bound degenerate clusters of $M<1.5 \times 10^{13} C_{\Lambda 0}^{1 / 2}$ ), but a size of $R_{\mathrm{J}} \approx 360 C_{\mathrm{F} 0}^{-1 / 6} \mathrm{kpc}$, smaller than the observed radii of typical clusters of galaxies. At $z=50$, the fermion mass of $\approx 50 \mathrm{~g}^{-1 / 4} \mathrm{eV}$ would lead to $M_{\mathrm{J}} \approx 10^{10} C_{\mathrm{F} 0}^{1 / 2} M_{\odot}$ and $R_{\mathrm{J}} \approx 7.9 C_{\mathrm{F} 0}^{-1 / 6} \mathrm{kpc}$; this result could be consistent with the dark halo of small spiral galaxies; however, for such a fermion mass, the redshift and the Jeans radius for $10^{11} M_{\odot}$ is $z \approx 235 C_{\mathrm{F} 0}^{-1 / 3}$ and $R_{\mathrm{J}} \approx 3.8 \mathrm{kpc}$.

Finally, we can insert Eq. (157) in (156); thus, we find

$\lambda_{\mathrm{J}}=\frac{2^{1 / 3} \pi^{8 / 3}}{3^{2 / 3} g^{2 / 3}} \frac{\left(\hbar c^{3}\right)^{2}}{G\left(m c^{2}\right)^{8 / 3}\left(M_{J} c^{2}\right)^{1 / 3}}$.

Comparing the Jeans radius, $R_{\mathrm{J}}=\lambda_{\mathrm{J}} / 2$, with the radius of a cluster of Newtonian degenerate fermions, $R_{\mathrm{c}}=5.1 b$, with $b$, given by Eq. (79), it can be seen that $R_{\mathrm{J}}=0.9 R_{\mathrm{c}}$. Therefore, the Jeans radius for Newtonian degenerate fermions is approximately the radius of a bound system of degenerate fermions.

\section{Conclusions}

We have considered structures composed of fully degenerate fermions in a background of dark energy assumed to be the cosmological constant. This has led to the determination of masses allowed for those clusters as a function of fermion mass; i.e., for each fermion mass, $m$, a minimum allowed cluster mass, $M_{m}$, and a maximum allowed cluster mass, $M_{M}$ have been deduced.

The minimum fermion mass that is able to build stable clusters of degenerate fermion is $0.024 g^{-1 / 4} \mathrm{eV}$, for which $M_{m}=$ $M_{M}$.

Clusters composed of degenerate fermions with masses smaller than $0.1 g^{-1 / 4} \mathrm{eV}$ are relativistic and simultaneously suffer the repulsive effects of the cosmological constant (for these masses, clusters with $M_{m}$ are relativistic and those with $M_{M}$ are affected by the cosmological constant).

We have shown that fermions of $m c^{2} \approx(15-30) g^{-1 / 4} \mathrm{eV}$ could build bound degenerate dark haloes with contributions to the rotational velocities as those needed to reproduce the rotational curves of galaxies. However, these fermions could not compose a degenerate dark halo of $10^{15} M_{\odot}$ with a size on the 
order of or greater than the visual radius of clusters of galaxies. Nevertheless, the dark halo in galaxy clusters could be composed of dark galaxies.

If degenerate dark clusters of masses much smaller than $10^{11} M_{\odot}$ were the dark haloes of dwarf galaxies, their radius should be much greater than those of degenerate clusters of $10^{11} M_{\odot}$. However, the tidal effects of larger neighboring galaxies would cause their sizes to be truncated and their masses to be reduced.

We saw that the dark halo of dwarf spheroidal galaxies cannot be modeled by degenerate fermions like those proposed here to describe the dark halo of typical galaxies. In fact, more compact self-gravitating spheres than those provided by degenerate fermions are necessary. Self-gravitating boson spheres might be an answer. In these systems, all bosons occupy the minimum Hartree orbital and the radius-mass relation of the cluster is of the type $R \sim M^{-1}$.

We showed that the values of rotational velocities of isolated small galaxies of $\sim 10 \mathrm{kpc}$ can be reproduced with dark clusters of masses similar to those proposed for typical galaxies, i.e., on the order of $\sim 10^{11} M_{\odot}$.

We found degeneracy conditions for fermions that are relativistic when they decouple from the radiation and for fermions that are non-relativistic at the decoupling. We saw that cold dark matter fermions cannot be fully degenerate.

We also derived the Jeans mass and radius of a cosmological background of Newtonian degenerate fermions as a function of the redshift. We saw that the Jeans masses and radii for degenerate fermions of $m c^{2} \approx(20-30) g^{-1 / 4} \mathrm{eV}$ at $z \approx 50$ are similar to the masses and radii derived (without considering any cosmological information) for the bound degenerate clusters that would be able to reproduce the rotation curves of galaxies. However, these degenerate fermions could not give rise to the dark halo of clusters of galaxies (a Jeans mass of about $10^{15} M_{\odot}$ would appear at $z \approx 10^{4}$, with a Jeans radius on the order of $1 \mathrm{kpc}$ ).

\section{References}

Assamagan, K., Brönnimann, Ch., Daum, M., et al. 1996, Phys. Rev. D, 53, 6065 Bell, E. F., \& de Jong, R. S. 2001, ApJ, 550, 212

Binney, J., \& Tremaine, S. 1987, Galactic Dynamics (Princeton: Princeton University Press)

Bond, J. R. 1988, in The Early Universe, eds. W. G. Unruh, \& G. W. Semenoff (Dordrecht: Reidel), Proc. NATO Adv. Study Inst., 283

Bothun, G. D., \& Thompson, I. B. 1988, AJ, 96, 877

Buskulic, D., Casper, D., de Bonis, I., et al. (ALEPH Collaboration) 1995, Phys. Lett. B, 349, 585

Caldwell, D. O. 1995, Nucl. Phys. B, 38, 394

Caldwell, R. R., Dave, R., \& Steinhardt, P. J. 1998, Phys. Rev. Lett., 80, 1582

Carr, B. J. 1994, ARA\&A, 32, 531

Castorina, E., Urbano, F., Massimiliano, L., et al. 2012, Phys. Rev. D, 86, 023517

Coble, K., Dodelson, S., \& Frieman, J. A. 1997, Phys. Rev. D, 55, 1851

Dekel, A., \& Silk, J. 1986, ApJ, 303, 39

de Vaucouleurs, G., \& Freeman, K. C. 1972, Vistas Astron., 14, 163

Diemand, J., Kuhlen, M., \& Madau, P. 2007, ApJ, 667, 859

Dolgov, A. D. 2002a, Phys. Rep., 370, 333

Dolgov, A. D., Hansen, S. H., Pastor, S., et al. 2002b, Nucl. Phys. B, 632, 363

Drimmel, R., \& Spergel, D. 2001, ApJ, 556, 181

Efstathiou, G. 1992, MNRAS, 256, 43

Efstathiou, G., Bridle, S. L., Lasenby, A. N., et al. 1999, MNRAS, 303, L47

Ferrara, A., \& Tolstoy, E. 2000, MNRAS, 313, 291

Freese, K., Kolb, E., \& Turner, S. 1983, Phys. Rev. D, 27, 1689

Glendenning, N. K. 1997, Compact Stars. Nuclear Physics, Particle Physics, and General Relativity (New York: Springer-Verlag)

Governato, F., Brook, C., Mayer, L., et al. 2010, Nature, 463, 203

Guo, Q., White, S., Li, C., \& Boylan-Kolchin, M. 2010, MNRAS, 404, 1111

Hamann, J., Lesgourgues, J., \& Mangano, G. 2008, JCAP, 0803, 004

Hoeft, M., Yepes, G., Gottlöber, S., \& Springel, V. 2006, MNRAS, 371, 401

Honma, M., \& Sofue, Y. 1996, PASJ, 45, L103

Jeans, J. H. 1919, Problems of Cosmology and Stellar Dynamics (Cambridge)

Kang, H., \& Steigman, G. 1992, Nucl. Phys. B, 372, 494
Kent, S. M. 1987, AJ, 93, 816

Kent, S. M., Dame, T. M., \& Fazio, G. 1991, ApJ, 378, 131

Kerins, E. J., \& Carr, B. J. 1994, MNRAS, 266, 775

Kim, S., Staveley-Smith, L., Dopita, M. A., et al. 1998, ApJ, 503, 674

Kippenhahn, R., \& Weigert, A. 1994, Stellar Structure and Evolution (New York: Springer-Verlag)

Klypin, A., Kravtsov, A. V., Valenzuela, O., \& Prada, F. 1999, ApJ, 522, 82

Klypin, A., Zhao, H., \& Somerville, R. S. 2002, ApJ, 573, 597

Koposov, S., Belokurov, V., Evans, N. W., et al. 2008, ApJ, 686, 279

Landau, L. D., \& Lifshitz, E. M. 2002, Statistical Physics, 5 Course of Theoretical Physics (Oxford: Butterworth-Heinemann)

Larson, R. B. 1974, MNRAS, 169, 229

Lattanzi, M., Ruffini, R., \& Vereshchagin, G. V. 2005, Phys. Rev. D, 72, 063003

Lee, J., \& Koh, I. 1996, Phys. Rev. D, 53, 2236

Lesgourgues, J., \& Liddle, A. R. 2001, MNRAS, 327, 13072

Lesgourgues, J., \& Pastor, S. 1999, Phys. Rev. D, 60, 103521

Lesgourgues, J., \& Pastor, S. 2006, Phys. Rept., 429, 307

Li, C., \& White, S. D. M. 2009, MNRAS, 398, 2177

Lobashev, V. M., Aseev, V. N., Beselev, A. I., et al. 1999, Phys. Lett. B, 460, 227

Martinez, G. D., Minor, Q. E., Bullock, J., et al. 2011, ApJ, 738, 55

Matos, T., \& Ureña-López, L. A. 2000, Class. Quant. Grav., 17, L75

Matos, T., \& Ureña-López, L. A. 2001, Phys. Rev. D, 63, 063506

Membrado, M., \& Aguerri, J. A. L. 1996, Int. J. Mod. Phys., 5, 257

Membrado, M., \& Pacheco, A. F. 2012, Europhys. Lett., 100, 39004

Membrado, M., Pacheco, A. F., \& Sañudo, J. 1989a, Phys. Rev. A, 39, 4207

Membrado, M., Abad, J., Pacheco, A. F., \& Sañudo, J. 1989b, Phys. Rev. D, 40, 2736

Moore, B., Ghigna, S., Governato, F., et al. 1999, ApJ, 524, L19

Moscoso, I. 1993, Nucl. Phys. B, 32, 365

Orito, M., Kajino, T., Mathews, G. J., et al. 2002, Phys. Rev. D, 65, 123504

Pacheco, A. F., Sañudo, J., \& Seguí, A. 1986, Phys. Lett B, 168, 223

Padmanabhan, T. 1995, Structure Formation in the Universe (Cambridge: Cambridge University Press)

Particle Data Group 2000, EPJ C, 15, 1

Passalacqua, L. 1997, Nucl. Phys. B, 55, 435

Pastor, S., \& Lesgourgues, J. 2000, Nucl. Phys. B, 81, 47

Peacock, S. 1999, Cosmological Physics (Cambridge: Cambridge University Press)

Pelupessy, F. I., van der Werf, P. P., \& Icke, V. 2004, A\&A, 422, 55

Perlmutter, S., Aldering, G., Deustua, S., et al. 1997, 191st AAS Meeting \#85.04, BAAS, 29, 1351

Perlmutter, S., Aldering, G., Goldhaber, G., et al. 1999, ApJ, 517, 565

Popa, L. A., \& Vasile, A. 2008, JCAP, 0806, 028

Raychaudhuri, A. K., Banerji, S., \& Banerjee, A. 1992, General Relativity, Astrophysics and Cosmology (New York: Springer-Verlag)

Sahni, V., \& Wang, L. 2000, Phys. Rev. D, 62, 103517

Sawala, T., Scannapieco, C., Maio, U., \& White, S. 2010, MNRAS, 402, 1599

Sawala, T., Guo, Q., Scannapieco, C., Jekins, A., \& White, S. 2011, MNRAS, 413, 659

Schmidt, B., Sunzeff, N. B., Phillips, M. M., et al. 1998, ApJ, 507, 46

Serpico, P. D., \& Raffelt, G. G. 2005, Phys. Rev. D, 71, 127301

Shapiro, S. L., \& Teukolsky, S. A. 1983, Black Holes, White Dwarfs, and Neutron Stars (New York: John Wiley \& Sons)

Shiraishi, M., Ichikawa, K., Ichiki, K., Sugiyama, N., \& Yamaguchi, M. 2009, JCAP, 0907, 005

Simon, J. D., \& Geha, M. 2007, ApJ, 670, 313

Simon, J. D., Geha, M., Minor, Q. E., et al. 2011, ApJ, 733, 46

Somerville, R. S. 2002, ApJ, 572, L23

Spergel, D. N., Verde, L., Peris, H., et al. 2003, ApJS, 148, 175

Springel, V., Wang, J., Vogelsberger, M., et al. 2008, MNRAS, 391, 1685

Steinhardt, P. J. 1996, Nature, 382, 768

Stinson, G. S., Dalcanton, J. J., Quinn, T., Kaufmann, T., \& Wadsley, J. 2007, ApJ, 667, 170

Strukov, I. A., Skulachev, D. P., \& Klypin, A. A. 1987, Large Scale of the Universe, IAU Symp. 130, eds. J. Audouze, M.-C. Pelletar, \& H. Szalay (Dordrecht: Kluwer), 93

Tollerud, E. J., Bullock, J. S., Strigari, L. E., \& Willman, B. 2008, ApJ, 688, 277

Tremaine, S., \& Gunn, J. E. 1979, Phys. Rev. Lett., 42, 407

Trimble, V. 1987, ARA\&A, 25, 425

Turner, M. S., \& White, M. 1997, Phys. Rev. D, 56, 4439

van der Marel, R. P., Alves, D. R., Hardy, E., \& Suntzeff, N. B. 2002, ApJ, 124, 2639

Wasserman, I., \& Salpeter, E. E. 1994, ApJ, 433, 670

Weinberg, S. 1972, Gravitation and Cosmology (New York: Wiley)

Weinheimer, Ch., Degen, B., Bleile, A., et al. 1999, Phys. Lett. B, 460, 219

Zaritsky, D. 1999, AJ, 118, 2824

Zavala, J., Jing, Y. P., Faltenbacher, A., et al. 2009, ApJ, 700, 1779 